\title{
Case studies of electrical characterisation of graphene by terahertz time-domain spectroscopy
}

Whelan, Patrick R.; Zhou, Binbin; Bezencenet, Odile; Shivayogimath, Abhay; Mishra, Neeraj; Shen, Qian; Jessen, Bjarke S.; Pasternak, Iwona; Mackenzie, David M.A.; Ji, Jie

Total number of authors:

37

Published in:

2D materials

Link to article, DOI:

$10.1088 / 2053-1583 / \mathrm{abdbcb}$

Publication date:

2021

Document Version

Publisher's PDF, also known as Version of record

Link back to DTU Orbit

Citation (APA):

Whelan, P. R., Zhou, B., Bezencenet, O., Shivayogimath, A., Mishra, N., Shen, Q., Jessen, B. S., Pasternak, I., Mackenzie, D. M. A., Ji, J., Sun, C., Seneor, P., Dlubak, B., Luo, B., Østerberg, F. W., Huang, D., Shi, H., Luo, D., Wang, M., ... Bøggild, P. (2021). Case studies of electrical characterisation of graphene by terahertz timedomain spectroscopy. 2D materials, 8(2), [022003]. https://doi.org/10.1088/2053-1583/abdbcb

\section{General rights}

Copyright and moral rights for the publications made accessible in the public portal are retained by the authors and/or other copyright owners and it is a condition of accessing publications that users recognise and abide by the legal requirements associated with these rights.

- Users may download and print one copy of any publication from the public portal for the purpose of private study or research.

- You may not further distribute the material or use it for any profit-making activity or commercial gain

- You may freely distribute the URL identifying the publication in the public portal 
TOPICAL REVIEW • OPEN ACCESS

Case studies of electrical characterisation of graphene by terahertz timedomain spectroscopy

To cite this article: Patrick R Whelan et al 2021 2D Mater. 8022003

View the article online for updates and enhancements. 
CrossMark

OPEN ACCESS

RECEIVED

29 October 2020

REVISED

20 November 2020

ACCEPTED FOR PUBLICATION

14 January 2021

PUBLISHED

17 February 2021

Original content from this work may be used under the terms of the Creative Commons Attribution 4.0 licence.

Any further distribution of this work must maintain attribution to the author(s) and the title of the work, journal citation and DOI.

TOPICAL REVIEW

\title{
Case studies of electrical characterisation of graphene by terahertz time-domain spectroscopy
}

Patrick R Whelan $^{1,2}$ (D), Binbin Zhou ${ }^{3}$, Odile Bezencenet ${ }^{4}$, Abhay Shivayogimath ${ }^{1,2}$ (D), Neeraj Mishra ${ }^{5,6}$, Qian Shen ${ }^{3,7,8}$, Bjarke S Jessen ${ }^{1,2,9,10}$, Iwona Pasternak ${ }^{11,12}$, David M A Mackenzie ${ }^{13}$ (D), Jie Ji ${ }^{1,2}$, Cunzhi Sun ${ }^{1,2}$, Pierre Seneor $^{14}$, Bruno Dlubak $^{14}$ (D) Birong Luo $^{15}$ (D), Frederik W Østerberg ${ }^{16}$, Deping Huang ${ }^{17}$, Haofei Shi ${ }^{17}$, Da Luo ${ }^{18}$, Meihui Wang ${ }^{18,19}$, Rodney S Ruoff ${ }^{18,19,20,21}$, Ben R Conran ${ }^{22}$, Clifford McAleese ${ }^{22}$, Cedric Huyghebaert ${ }^{23}$ D , Steven Brems ${ }^{23}$, Timothy J Booth ${ }^{1,2}$, Ilargi Napal ${ }^{24}$, Wlodek Strupinski ${ }^{11,12}$, Dirch H Petersen $^{25}$, Stiven Forti ${ }^{5}$ (D), Camilla Coletti ${ }^{5,6}$ (D), Alexandre Jouvray ${ }^{22}$, Kenneth B K Teo ${ }^{22}$, Alba Centeno $^{24}\left(\mathbb{D}\right.$, Amaia Zurutuza ${ }^{24}$, Pierre Legagneux ${ }^{4}$, Peter U Jepsen ${ }^{2,3}$ and Peter Bøggild ${ }^{1,2}$

1 DTU Physics, Technical University of Denmark, Fysikvej, Bld. 309, DK-2800 Kgs. Lyngby, Denmark

2 Centre for Nanostructured Graphene (CNG), Technical University of Denmark, Ørsteds Plads 345C, DK-2800 Kgs. Lyngby, Denmark

3 DTU Fotonik, Technical University of Denmark, Ørsteds Plads 343, DK-2800 Kongens Lyngby, Denmark

4 Thales Research and Technology, 91767 Palaiseau, France

5 CNI@NEST, Istituto Italiano di Tecnologia, Piazza San Silvestro 12, 56127 Pisa, Italy

6 Graphene Labs, Istituto Italiano di Tecnologia, Via Morego 30, 16163 Genova, Italy

7 School of Information Engineering, Nanchang University, Nanchang 330031, People's Republic of China

8 College of Science, Zhejiang University of Technology, Hangzhou 310014, People's Republic of China

9 Department of Physics, Columbia University, New York, NY 10027, United States of America

10 Department of Mechanical Engineering, Columbia University, New York, NY 10027, United States of America

11 Faculty of Physics, Warsaw University of Technology, Koszykowa 75, 00-662 Warsaw, Poland

12 Vigo System S.A., 129/133 Poznanska Str, 05-850 Ozarow Mazowiecki, Poland

13 Department of Electronics and Nanoengineering, Aalto University, Box 13500, FI-00076 Aalto, Finland

14 Unité Mixte de Physique, CNRS, Thales, Université Paris-Saclay, 91767 Palaiseau, France

15 College of Physics and Materials Science, Tianjin Normal University, 300387 Tianjin, People's Republic of China

16 CAPRES-A KLA Company, Scion-DTU, Bld. 373, DK-2800 Kgs. Lyngby, Denmark

17 Chongqing Institute of Green and Intelligent Technology, Chinese Academy of Sciences, 266 Fang Zheng Ave., Chongqing 400714, People's Republic of China

18 Center for Multidimensional Carbon Materials (CMCM), Institute for Basic Science (IBS), Ulsan 44919, Republic of Korea

19 Department of Chemistry, Ulsan National Institute of Science and Technology (UNIST), Ulsan 44919, Republic of Korea

20 Department of Materials Science and Engineering, Ulsan National Institute of Science and Technology (UNIST), Ulsan 44919, Republic of Korea

21 School of Energy and Chemical Engineering, Ulsan National Institute of Science and Technology (UNIST), Ulsan 44919, Republic of Korea

22 AIXTRON Ltd, Buckingway Business Park, Anderson Road, Swavesey, Cambridge CB24 4FQ, United Kingdom

23 IMEC vzw, Kapeldreef 75, BE-3001 Leuven, Belgium

24 Graphenea Semiconductor S.L.U, Paseo Mikeletegi 83, 20009 San Sebastian, Spain

25 DTU Energy, Technical University of Denmark, Fysikvej, Bld. 310, DK-2800 Kgs. Lyngby, Denmark

E-mail:pbog@dtu.dk

Keywords: terahertz spectroscopy, large-scale graphene, CVD graphene, electrical mapping

\begin{abstract}
Graphene metrology needs to keep up with the fast pace of developments in graphene growth and transfer. Terahertz time-domain spectroscopy (THz-TDS) is a non-contact, fast, and non-destructive characterization technique for mapping the electrical properties of graphene. Here we show several case studies of graphene characterization on a range of different substrates that highlight the versatility of THz-TDS measurements and its relevance for process optimization in graphene production scenarios.
\end{abstract}

\section{Introduction}

Large-area, fast, non-destructive, and precise measurement techniques are required as the manufacturing of graphene and graphene technology is scaled up [1]. Rapid developments in graphene growth and transfer demand improved quality control during fabrication. Spatially determining the electrical parameters of a given sample is critical, since electrical inhomogeneities may 
dramatically reduce the performance of final devices.

Terahertz time-domain spectroscopy (THz-TDS) is a non-contact and non-destructive measurement technique that can spatially map the electrical properties (DC conductivity $\sigma_{\mathrm{DC}}$, scattering time $\tau$, carrier density $n$, and mobility $\mu$ ) of graphene on substrates relevant for the use of graphene such as $\mathrm{Si}$ [2-6], quartz [7], SiC [8, 9], sapphire [10], and polymers $[11,12]$, and is maturing as a metrology tool for characterizing the electrical properties of graphene $[1,13-16]$. $\mathrm{THz}$ spectroscopy is also applicable for measuring conductivity and scattering processes of other 2D materials such as $\mathrm{MoS}_{2}, \mathrm{WSe}_{2}$, and VN [17-20].

Here, we evaluate $\mathrm{THz}$-TDS for characterization of graphene in a wide range of different scenarios. This includes optimization of graphene growth, transfer, and post-transfer processing. We will also discuss the possible implementation of real-time THz-TDS in both batch and roll-to-roll (R2R) production of graphene.

The paper is organized as follows. In section 2 we discuss how to perform THz-TDS measurements of graphene and how to analyze THz-TDS data in different cases. Furthermore, we present how to extract electrical and electronic parameters $\left(\sigma_{\mathrm{DC}}, \tau, \mathrm{n}, \mu\right.$, renormalized Fermi velocity $\nu_{\mathrm{F}}^{*}$ ) by fitting the frequency dependent conductivity at $\mathrm{THz}$ frequencies with the Drude model. In section 3 we present several case studies of $\mathrm{THz}$-TDS measurements of graphene including encapsulated graphene and $\mathrm{THz}$-TDS for quality control in graphene production scenarios.

\section{Methods}

THz-TDS measurements were performed with two different setups. The main setup is a commercial fibre-coupled spectrometer (Picometrix T-Ray 4000) with a programmable stage that allows for raster scanning samples in the focal plane of the $\mathrm{THz}$ beam and creating spatial maps of the electrical properties of graphene [14]. The setup is capable of measuring within a frequency range of $\sim 0.5-1.5 \mathrm{THz}$ with a spot size of $\sim 400 \mu \mathrm{m}$ at $1 \mathrm{THz}$ [5]. Measurements were performed with this setup and in transmissionmode if not elsewise stated. The second setup is a custom-built ultra-broadband setup based on twocolor femtosecond air-plasma $\mathrm{THz}$ generation and an air biased coherent detection scheme [21-23]. This setup covers an extended spectral frequency range up to $\sim 30 \mathrm{THz}$ due to the extremely short pulse duration of a few tens of fs compared to the few-ps duration for the commercial setup as shown in figure 1. For the high-resistivity (HR, $\rho>10 \mathrm{k} \Omega \mathrm{cm}$ ) silicon substrates used, the spectral range of reproducible results is limited to approximately $2-10 \mathrm{THz}$.

\subsection{THz-TDS measurement schemes}

THz-TDS measurements can be performed in transmission mode or reflection mode as illustrated in figures 1(a)-(c) with examples of measured $\mathrm{THz}$ time-domain waveforms for graphene on different substrates shown in figure 1(d). For transmission mode measurements it is a requirement that the substrate is at least partially transparent to $\mathrm{THz}$ radiation, with $\mathrm{HR} \mathrm{Si}, \mathrm{SiC}$, sapphire, and many polymeric materials having been successfully used. The waveforms typically contain several transients that are separated in time, due to internal reflections inside the substrate; a transient $\tilde{E}^{(\mathrm{dir})}$ from the directly transmitted pulse followed by a transient $\tilde{E}^{(1 s t)}$ from the first internal reflection inside the substrate, a transient $\tilde{E}^{(2 n d)}$ from the second internal reflection and so forth. The internal reflections are easily seen in figure $1(\mathrm{~d})$ when HR-Si is used as substrate, while for $\mathrm{SiC}$ these internal reflections are not as clear due to the higher absorption in the substrate compared to HR-Si [24, 25]. For thin (up to $400 \mu \mathrm{m}$ ) polymeric substrate (such as polyethylene terephthalate, PET) the transients from directly transmitted pulse and internal reflections may appear as a single reshaped transient, since the thickness and refractive index of the substrate leads to a propagation time within the substrate that is shorter than the $\mathrm{THz}$ pulse duration [11]. Due to the short pulse duration of the custombuilt air plasma setup it is still possible to measure individual transients for polymer substrates with such a setup [12].

The Fourier transforms of the $\mathrm{THz}$ waveforms transmitted through substrate $\tilde{E}_{\text {sub }}(\omega)$ and graphene covered substrate $\tilde{E}_{\text {film }}(\omega)$ are used to calculate the frequency-dependent complex transmission function $\tilde{T}_{\text {film }}(\omega)=\tilde{E}_{\text {film }}(\omega) / \tilde{E}_{\text {sub }}(\omega)$, from which the complex sheet conductivity of the graphene layer, $\tilde{\sigma}_{s}(\omega)=\sigma_{1}+i \sigma_{2}$ is obtained. The transmission function is calculated for individual transients by applying window functions. For data acquired with the commercial spectrometer we generally use a cosine tapered (Tukey) window function with $50 \%$ taper length (0.5 cosine fraction) and a $2.5 \mathrm{ps}$ width. The window function width and tapering were determined by varying the parameters until small changes did not induce significant re-shaping of the frequency-dependent conductivity.

The means of determining $\tilde{\sigma}_{s}(\omega)$ from $\tilde{T}_{\text {film }}(\omega)$ depends on the transient used for the analysis [14]. For the directly transmitted pulse and the first internal reflection the relations are, respectively,

$$
\tilde{\sigma}_{\mathrm{s}}^{(\mathrm{dir})}(\omega)=\frac{n_{\mathrm{A}}}{Z_{0}}\left(\frac{1}{\tilde{T}_{\text {film }}^{(\operatorname{dir})}(\omega)}-1\right)
$$


a

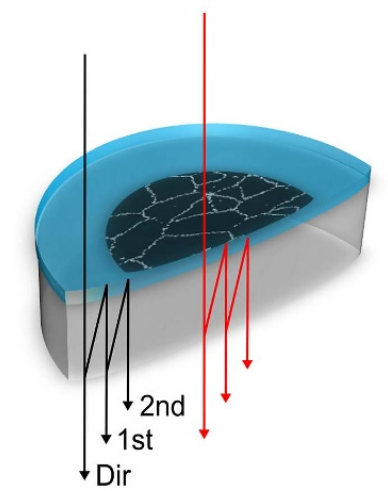

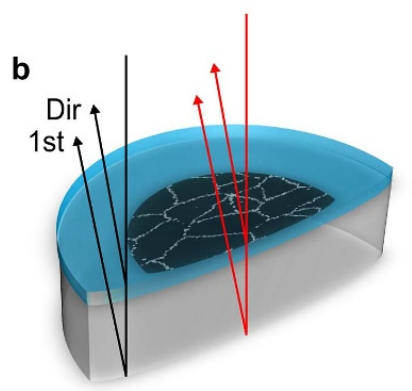

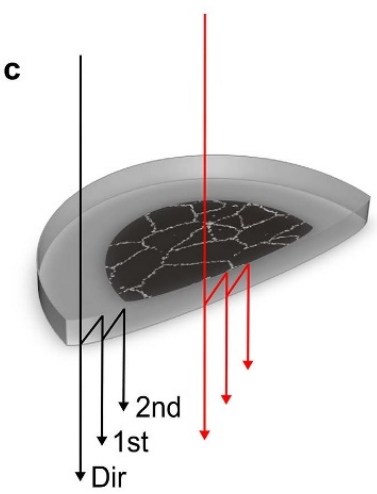

d

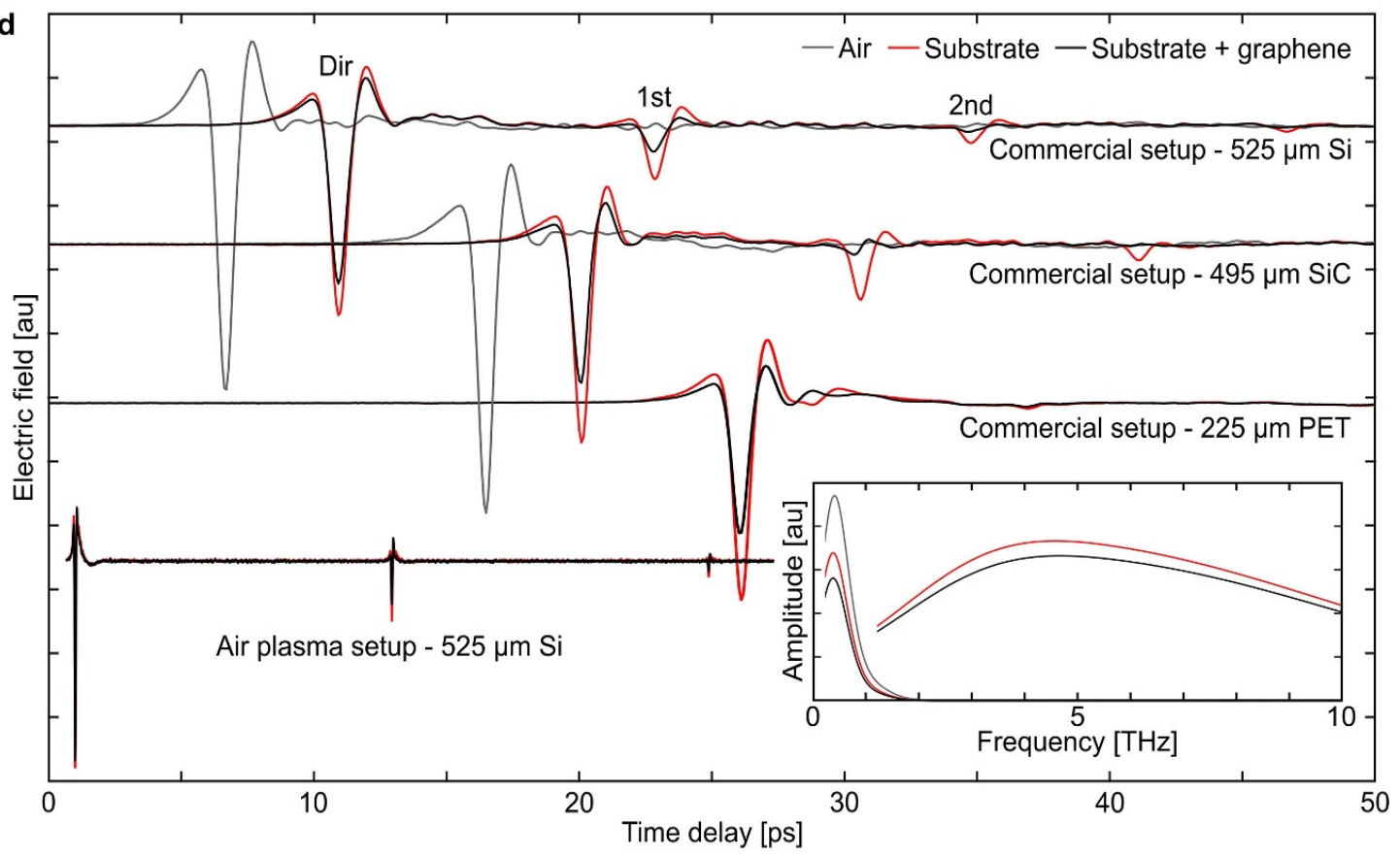

Figure 1. (a)-(c) Illustrations of THz-TDS measurements, (a) transmission mode, (b) reflection mode, and (c) transmission mode for thin substrate. Arrows highlight internal reflections within substrate. (d) Time-domain waveforms acquired in transmission mode for graphene on different substrates with commercial and air plasma setup. Inset shows Fourier transforms of the part of the waveform belonging to the first internal reflection for measurements on $525 \mu \mathrm{m}$ Si with the commercial and air plasma setups.

$$
\tilde{\sigma}_{\mathrm{s}}^{(1 \mathrm{st})}(\omega)=\frac{n_{\mathrm{A}} \sqrt{n_{\mathrm{A}}^{2}+4 n_{\mathrm{A}} n_{\mathrm{B}} \tilde{T}_{\text {film }}^{(1 \mathrm{st})}(\omega)+4 n_{\mathrm{B}}^{2} \tilde{T}_{\text {film }}^{(1 \mathrm{st})}(\omega)}-n_{\mathrm{A}}^{2}-2 n_{\mathrm{A}} n_{\mathrm{B}} \tilde{T}_{\text {film }}^{(1 \mathrm{st})}(\omega)}{2 n_{\mathrm{B}} Z_{0} \tilde{T}_{\text {film }}^{(1 \mathrm{st})}(\omega)}
$$

where $n_{\mathrm{A}}=\tilde{n}_{\text {sub }}+1$ and $n_{\mathrm{B}}=\tilde{n}_{\text {sub }}-1$ for a substrate with refractive index $\tilde{n}_{\text {sub }}$ and $Z_{0}$ is the vacuum impedance $[14,26]$. The Tinkham relations are used for transmission and reflection coefficients of thin conducting films $\left(d_{\text {film }} \ll \lambda / n_{\text {film }}\right)$ [27].

In the case of thin polymeric substrates, where $\tilde{T}_{\text {film }}(\omega)$ contains terms from all transmitted transients, $\sigma_{\mathrm{s}}(\omega)$ can be determined as

$$
\tilde{\sigma}_{\mathrm{s}}^{\text {(all })}(\omega)=\frac{n_{\mathrm{A}}^{2}-\tilde{p}^{2} n_{\mathrm{B}}^{2}\left(\tilde{p}^{2} n_{\mathrm{B}}^{2}-n_{\mathrm{A}}^{2}\right) \tilde{T}_{\mathrm{film}}(\omega)}{\left(n_{\mathrm{A}}+\tilde{p}^{2} n_{\mathrm{B}}\right) Z_{0} \tilde{T}_{\mathrm{film}}(\omega)}
$$

where $\tilde{p}=\exp \left(-i \omega d_{\mathrm{sub}} n_{\mathrm{sub}} / c\right) \quad$ represents the propagation term in a substrate with thickness $d_{\text {sub }}[11]$.

Recently, it has been shown that it is also possible to perform reference-free extraction of dielectric and 
electrical properties of substrates and thin conducting films from THz-TDS measurements [28, 29]. This is achieved by comparing individual transients from only the sample waveform, which means that the reference measurement can be omitted and furthermore decreases errors from signal fluctuations originating from the measurement setup such as timing jitter [26], periodic sampling errors [30], and uneven stage movement. From the directly transmitted pulse and the first internal reflection, $\tilde{\sigma}_{\text {(ref-free })}(\omega)$ is determined from $\tilde{T}_{\text {film }}^{\text {(ref-free) }}(\omega)=\tilde{E}_{\text {film }}^{(1 \mathrm{st})}(\omega) / \tilde{E}_{\text {film }}^{(\text {dir })}(\omega)$ as [29]

$$
\tilde{\sigma}_{\mathrm{s}}^{\text {(ref-free) }}(\omega)=\frac{n_{\mathrm{B}}^{2} \Delta \tilde{p}-n_{\mathrm{A}}^{2} \tilde{T}_{\text {film }}^{\text {(ref-free) }}(\omega)}{Z_{0}\left(n_{\mathrm{A}} \tilde{T}_{\text {film }}^{(\text {ref-free })}(\omega)+n_{\mathrm{B}} \Delta \tilde{p}\right)},
$$

where $\Delta \tilde{p}=\exp \left(-2 i \omega d_{\text {sub }} n_{\text {sub }} / c\right) \exp \left(-i \emptyset_{\mathrm{G}}\right) \exp \left(d_{\text {sub }}\right.$ $\alpha$ ). Compared to $\tilde{p}$ in equation (3), the second term $\exp \left(-i \emptyset_{\mathrm{G}}\right)$ is a correction for the Gouy phase shift $\emptyset_{\mathrm{G}}$ [31], and the last term $\exp \left(d_{\mathrm{sub}} \alpha\right)$ is an amplitude correction based on the absorption coefficient $\alpha$ of the substrate. These two additional terms are not necessary in equation (3) since they are equal for $\tilde{E}_{\text {film }}(\omega)$ and $\tilde{E}_{\text {sub }}(\omega)$ and cancel out. It is possible to determine $d_{\text {sub }}$ from the distance between peaks in frequency domain from the Fourier transform of the full $\mathrm{THz}$ waveform including all transients. In practice, the correction terms for the amplitude and Gouy phase shift can be determined from one single calibration-like measurement [29]. This is advantageous compared to the standard referenced analysis in equations (1)-(3), where reference measurements must be acquired frequently to minimize effects from environment, mechanical stage movement and $\mathrm{THz}$ source fluctuations, all of which have no influence on the reference-free analysis. Also, at lower frequencies, where commercial spectrometers are operating, it is possible to extract meaningful electrical parameters for graphene on non-absorbing substrates without having to correct for amplitude and Gouy phase shift [29].

\subsection{Conductivity analysis of THz-TDS spectra}

The optical conductivity of graphene in the $\mathrm{THz}$ frequency range is mainly determined by intraband carrier transitions and the measured frequencydependent sheet conductivity of graphene is well described by the Drude model [2-4, 6, 7, 14, 32, 33]

$$
\tilde{\sigma}_{s}(\omega)=\frac{\sigma_{\mathrm{DC}}}{1-i \omega \tau}
$$

where $\sigma_{\mathrm{DC}}=1 / \rho_{\mathrm{s}}$ is the conductivity at $\omega=0$. Figure 2(a) shows an example for graphene on $\mathrm{SiC}$ $[9,34]$ where $\tilde{\sigma}_{\mathrm{s}}^{\text {dir }}(\omega)$ is fitted with the Drude model to obtain $\sigma_{\mathrm{DC}}$ and $\tau$. From semiclassical Boltzmann transport theory $[14,35]$ the calculation of $n$ and $\mu$ from $\sigma_{\mathrm{DC}}$ and $\tau$ follows as $[6,9,12]$

$$
\begin{array}{r}
n=\frac{\pi \hbar^{2}}{e^{4} \nu_{\mathrm{F}}^{2}}\left(\frac{\sigma_{\mathrm{DC}}}{\tau}\right)^{2}, \\
\mu=\frac{e^{3} \nu_{\mathrm{F}}^{2}}{\pi \hbar^{2}} \frac{\tau^{2}}{\sigma_{\mathrm{DC}}},
\end{array}
$$

where $\nu_{\mathrm{F}}$ is the Fermi velocity. While $\nu_{\mathrm{F}}$ is often taken as a constant, it is necessary to renormalize $\nu_{\mathrm{F}}$ $\left(\nu_{\mathrm{F}}^{*}\right)$ due to interacting electrons in graphene [36-39], which are greatly affected by the dielectric environment, and thus the substrate. The square dependence of $\mathrm{n}, \mu$ on $\nu_{\mathrm{F}}$ in equations (6) and (7) highlights the importance of using the correct value for this parameter. The renormalization of $\nu_{\mathrm{F}}$ is carried out for THz-TDS measurements of graphene [12], by determining $\nu_{\mathrm{F}}^{*}$ as [39]

$$
\frac{\nu_{\mathrm{F}}^{*}}{\nu_{\mathrm{F}}}=1+C(\alpha) \alpha \ln \left(\Lambda / k_{\mathrm{F}}\right)
$$

where $\Lambda=1.75 \AA^{-1}, k_{\mathrm{F}}=\sqrt{\pi n}, C(\alpha)=(4(1+(\pi / 2)$ $\alpha))^{-1}$ and $\alpha=e^{2} /\left(4 \pi \hbar \nu_{\mathrm{F}} \varepsilon \varepsilon_{0}\right)$ [39]. We use $\nu_{\mathrm{F}}=0.85 \times 10^{6} \mathrm{~m} \mathrm{~s}^{-1}$ [36] and determine the effective permittivity $\varepsilon$ as $\varepsilon=\left(\varepsilon_{\mathrm{s}}+1\right) / 2$ using relative permittivity $\varepsilon_{\mathrm{s}}=4.4$ for $\mathrm{SiO}_{2}$ substrate [40]. In practice, $\mathrm{n}, \mu, \nu_{\mathrm{F}}^{*}$ are determined by iteratively solving equations (6) and (8) for $n$ and $\nu_{\mathrm{F}}^{*}$ and finally calculating $\mu$ from equation (7) based on the obtained value for $\nu_{\mathrm{F}}^{*}$.

The electrical parameters can also be extracted from reflection mode THz-TDS measurements [41] and a comparison between the conductivity extracted from transmission and reflection mode THz-TDS has also shown to yield similar results [42]. Reflection mode THz-TDS measurements of graphene will not be described in further detail here but have been described elsewhere [14, 41-44].

Complementary measurements have been performed in many cases in order to validate the extracted electrical properties of graphene measured by THz-TDS [14]. The measurements conducted for comparison with $\mathrm{THz}$-TDS varies across a range of characterization methods including optical microscopy [29, 45], Hall measurements [6, 9, 12, 46], Raman spectroscopy [5, 8, 41], Kelvin probe force microscopy [8, 47], and micro four-point probe (M4PP) measurements [5, 8, 33, 48]. M4PP is a unique metrology technology developed by DTU and Capres A/S where the conductance is measured across a conducting wafer or thin film, using a 12-point microfabricated probe, with a probe pitch down to $1 \mu \mathrm{m}[5,14,33,49-52]$. By combining the information from multiplexed four-terminal measurements (using different combinations of the 12 individual cantilever probes) according to specific protocols [48], values for the electrical properties can be extracted with far higher accuracy than ordinary M4PP measurements. 

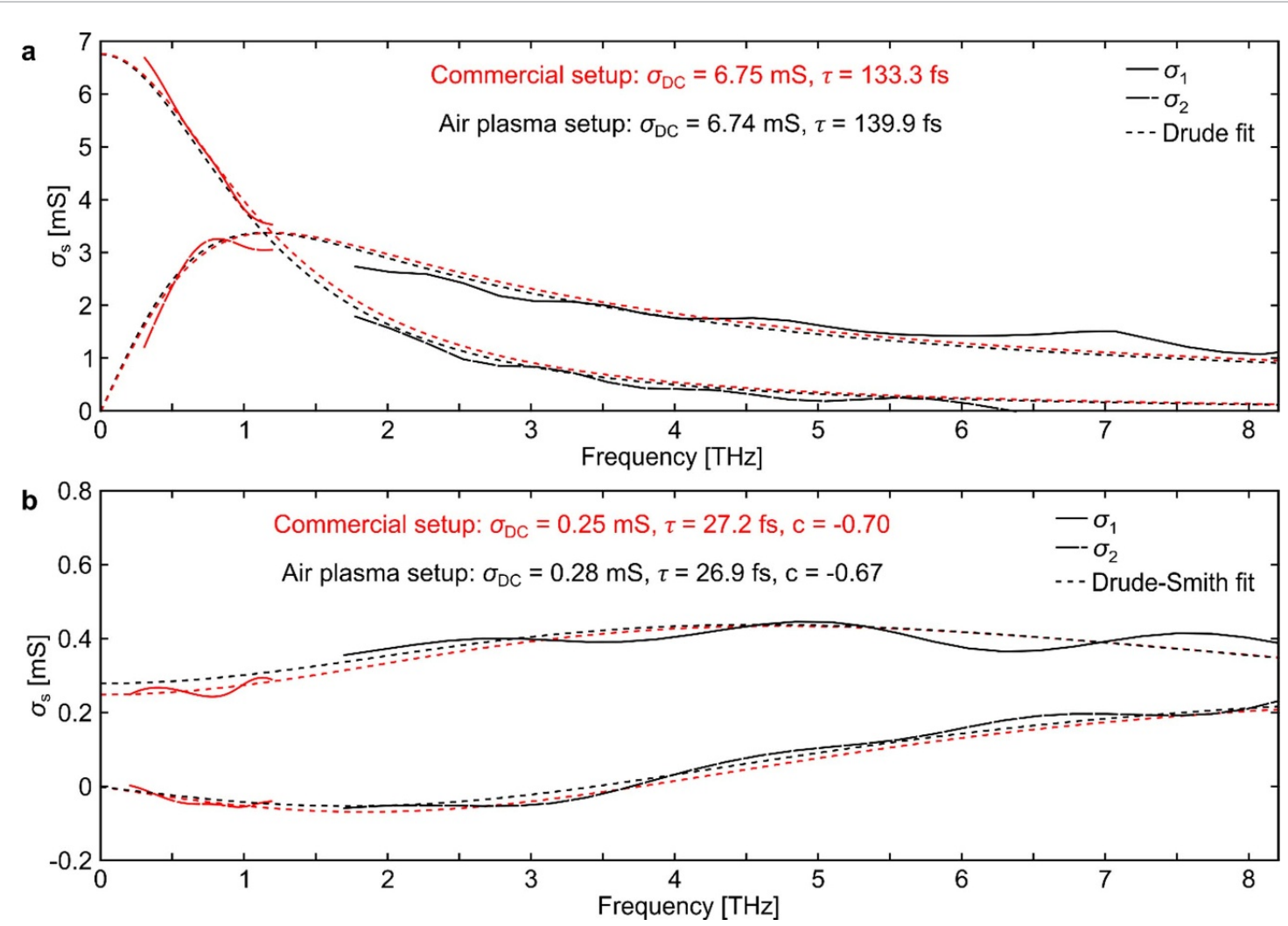

Figure 2. Comparison of THz-TDS frequency-dependent conductivity measured with commercial and air plasma setup. (a) Graphene on SiC fitted with the Drude model. (b) Graphene grown on Ge and transferred to HR-Si fitted with the Drude-Smith model.

\subsubsection{Non-uniform graphene}

In some instances, $\tilde{\sigma}_{\mathrm{s}}(\omega)$ does not appear to follow the Drude model but instead at low frequencies shows a reduction in the real part of the conductivity while the imaginary part of the conductivity becomes negative as seen in figure 2(b). Such conductivity behavior has previously been reported for semiconductor nanostructures and thin metallic films at the metal-insulator percolation threshold [15, 53-57], and is characteristic of systems where the charge carriers experience some degree of preferential backscattering in addition to the isotropic scattering assumed in the Drude model. As the distance between line defects approaches the mean free path (average distance a charge carrier can travel until its initial velocity is randomized by scattering processes), the complex conductivity becomes increasingly difficult to describe with the Drude model.

For 2D materials, such behavior can originate from any line-shaped defect, including damage occurring in the transfer process (tears, rips, folds, wrinkles), lithographically defined edges (i.e. in arrays or gratings) but most commonly from grain boundaries that are present in nearly all graphene grown by chemical vapor deposition, as well as by most other synthesis methods [58]. Grain boundaries occur as crystal domains from multiple nucleation points coalesce to form a continuous film. At the grain boundary itself, the stitching of neighboring, rotated domains is typically facilitated by introduction of a line of 5-7 defects, which are more densely spaced for large grain misalignment angles [59]. Moreover, the momentum mismatch between misaligned neighboring grains contributes to the energy barrier that leads to reflection and carrier localization.

The conductivity response has in these cases been described by the phenomenological Drude-Smith model developed for systems with some degree of carrier localization $[60,61]$. The Drude-Smith model is expressed to first order as [60]

$$
\tilde{\sigma}_{\mathrm{s}}(\omega)=\frac{W_{\mathrm{D}}}{1-i \omega \tau}\left(1+\frac{c}{1-i \omega \tau}\right),
$$

where $W_{\mathrm{D}}$ is the Drude weight $\left(\sigma_{\mathrm{DC}}=W_{\mathrm{D}}(1+c)\right)$ and the parameter $c(-1 \leqslant c \leqslant 0)$ describes the degree of preferential backscattering. If $c=0$, the ensemble carrier momentum is relaxing exponentially on a timescale of $\tau$, which in the Drude model is also the carrier scattering time. In the Drude model, every scattering event randomizes the momentum. In contrast, during $\mathrm{THz}$ measurements all carriers are backscattered during one cycle of the AC-field for $c=-1$ (complete suppression of DC conductivity). The Drude-Smith model has previously been applied to graphene in cases where the homogeneity of the graphene layer was suspected to be low $[33,62]$ and also provides a good fit to $\tilde{\sigma}_{s}(\omega)$ in figure $2(\mathrm{~b})$ for 
graphene grown on $\mathrm{Ge}[63,64]$ and transferred to HR-Si [65]. We note that due to the phenomenological nature of the Drude-Smith model, it is not clear how the extracted scattering time should be interpreted, whether macroscopic carrier mobility can be extracted and what exactly the physical meaning of a non-zero backscatter parameter means. Alternative interpretations of the Drude-Smith model as well as alternative models are therefore being developed and discussed in literature $[15,66-70]$.

Figure 2 shows Drude and Drude-Smith model fits to measurements carried out with the commercial as well as the air plasma setup, see section 2.1. With its much broader frequency range the air plasma setup allows us to extend and verify the values extracted for $\sigma_{\mathrm{DC}}, \tau$, and $c$ with the commercial setup, due to the greater accuracy of the fits to the broader spectrum. As seen in figure 2 we obtain similar values from both setups, which has also been shown for different cases of graphene on HR-Si [33] and PET [12].

Both the Drude and Drude-Smith behavior is successfully described using classical Monte Carlo simulations [69]. Here the motion of carriers subject to isotropic (Drude) and boundary scattering (preferential backscattering) is calculated to generate conductivity spectra that can be directly compared to conductivity spectra extracted from THz-TDS measurements. We employ a strategy inspired by the impulse response approach [69] where an ensemble of carriers (travelling at a constant Fermi velocity $\nu_{\mathrm{F}}$ ) are designated an initial finite average momentum, corresponding to a drift velocity obtained for a constant electrical field and a certain carrier mobility. In the absence of the electrical field, scattering on isotropically scattering defects corresponding to a certain mean free path $\ell_{\mathrm{mfp}}$ and carrier mobility $\mu$ as well as scattering on boundaries (with reflection coefficient, $0<R \leq 1$ ) causes the average current to decrease towards zero over time. The frequency-dependent conductivity is obtained by Fourier-transforming the time-dependent current $I(t)$ extracted from averaging over $10^{5}-10^{6}$ trajectories. In figure 3 we show the simulated conductivity spectra for graphene with a carrier mobility of $2150 \mathrm{~cm}^{2} \mathrm{~V} \mathrm{~s}^{-1}$ and a carrier density of $10^{12} \mathrm{~cm}^{-2}$, which are typical numbers for CVD graphene on a $\mathrm{SiO}_{2}$ substrate. The resulting mean free path $\ell_{\mathrm{mfp}}=$ $25 \mathrm{~nm}$, can be found from the Boltzmann transport equation $\sigma_{\mathrm{DC}}=\left(2 e^{2} / h\right) k_{\mathrm{F}} \ell_{\mathrm{mfp}}$ and $\sigma_{\mathrm{DC}}=n e \mu$. A network of partially $(0<R<1$, figure $3(\mathrm{a}))$ or fully reflecting $(R=1$, figure $3(\mathrm{~b})$ ) boundaries mimics a grain boundary landscape with average grain sizes of $125 \mathrm{~nm}, 250 \mathrm{~nm}, 500 \mathrm{~nm}$ and up to $4000 \mathrm{~nm}$, which can be compared to the boundary-free Drude conductivity (black lines). For $R=1$ (figure 3(b)), domain sizes up to $4 \mu \mathrm{m}$ exhibit the depression of the DC conductivity characteristic of preferential backscattering, despite the grain size being 160 times larger than the mean free path. This shows that carrier localization effects can be present for much lower line defect densities (or grain sizes in the case of polycrystalline graphene) than anticipated in our previous work [33], indicating that THz-TDS may be helpful in analyzing the microstructure of graphene films. More work needs to be done, however, to accurately determine the device carrier mobility for small-grain graphene films with strong non-Drude-like behavior, which may be particular prevalent for direct CVD growth on substrates like $\mathrm{SiC}[7,8], \mathrm{SiO}_{2}$ and sapphire[1] where grain sizes are typically smaller than on copper substrates, as well as high-throughput R2R CVD reactors.

\section{Case studies}

In the following we present different case studies of THz-TDS measurements of graphene. First, we show that values for $\sigma_{\mathrm{DC}}$ extracted from the Drude-Smith model agree well with reference measurement across 4 inch silicon wafer substrates covered with graphene. The remaining cases includes a highlight of recent developments in reference-free analysis and examples showing the relevance of THz-TDS in various graphene production and optimization scenarios.

\subsection{Graphene conductivity from the Drude-Smith model}

Values of $\sigma_{\mathrm{DC}}$ extracted from THz-TDS conductivity spectra following the Drude model have been validated several times against Hall and M4PP measurements $[6,8,11,33,47]$. There is however comparatively limited data available $[62,71]$ for measurements of graphene when $\tilde{\sigma}_{s}(\omega)$ does not appear to follow the Drude model and in particular for measurements at 4 inch wafer-scale.

A map of $\sigma_{\mathrm{DC}}$ extracted from the Drude-Smith model for a 4 inch wafer covered with graphene (from Graphenea, Spain) measured by THz-TDS is shown in figure 4(a). The sample shows a base conductivity of $\sim 2 \mathrm{mS}$ with clearly defined lines of lower conductivity. The origin of these lines is not clear. A high-resolution optical map of the sample does not reveal any distinct non-uniformities, such as tears and scratches in the graphene layer. Figure 4 shows values of $c$ and $\sigma_{\mathrm{DC}}$ from THz-TDS together with the sheet conductance $\left(G_{s}\right)$ from M4PP following a line scan across the wafer with $1 \mathrm{~mm}$ step size. The M4PP measurements were performed with $10 \mu \mathrm{m}$ probe pitch on a CAPRES microRSP-A300 measurement system using a newly developed sub-probe analysis for conductivity retrieval [48]. The values of $\sigma_{\mathrm{DC}}$ and $G_{\mathrm{s}}$ are in reasonable agreement, and show similar, distinct variations at positions matching the low conductivity lines in the wafer map. This indicates that THz-TDS can be used for large-area conductivity maps, even when the individual conductivity 

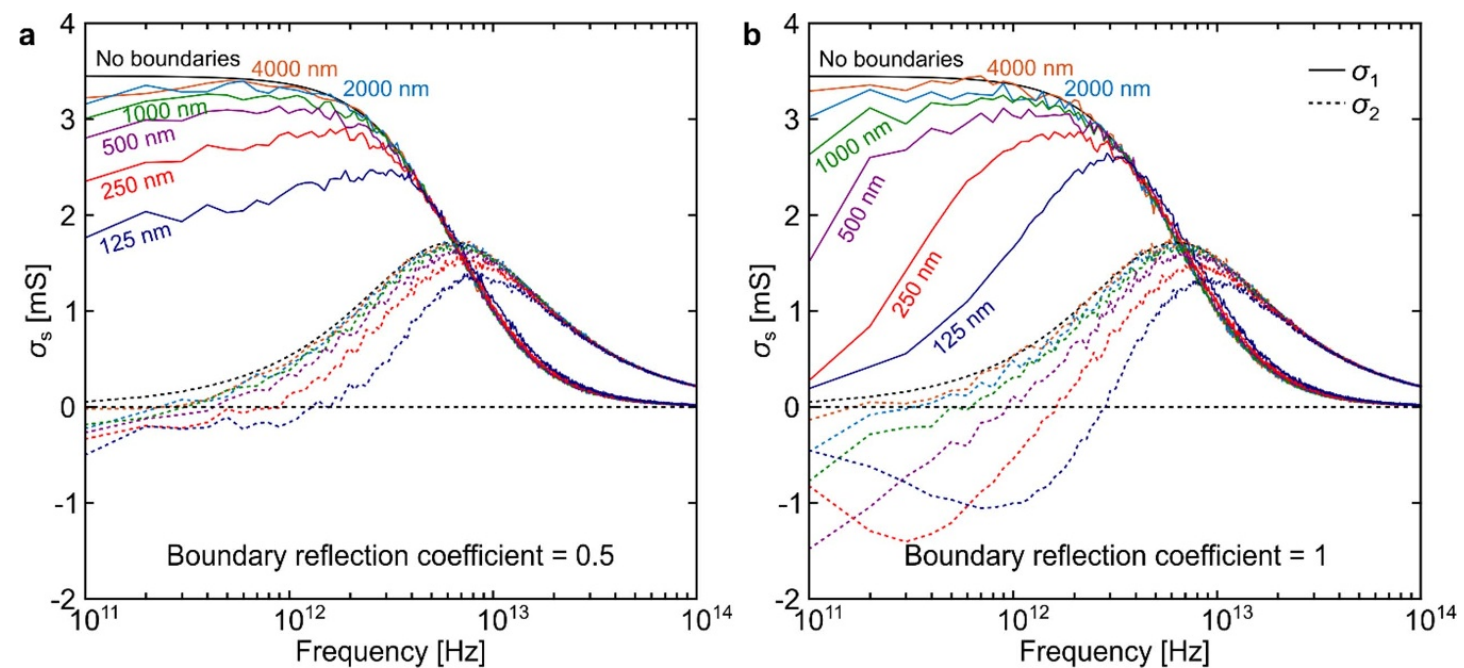

Figure 3. Monte Carlo simulated conductivity spectra for graphene with average grain boundary size from $125 \mathrm{~nm}$ and up to $4000 \mathrm{~nm}$ and a mean free path of $25 \mathrm{~nm}$, for boundary reflection coefficient (a) $R=0.5$, (a) $R=1$. Full lines indicate the real part $\sigma_{1}$ and dashed lines indicate the imaginary part $\sigma_{2}$ of the complex sheet conductivity $\sigma_{\mathrm{s}}$.
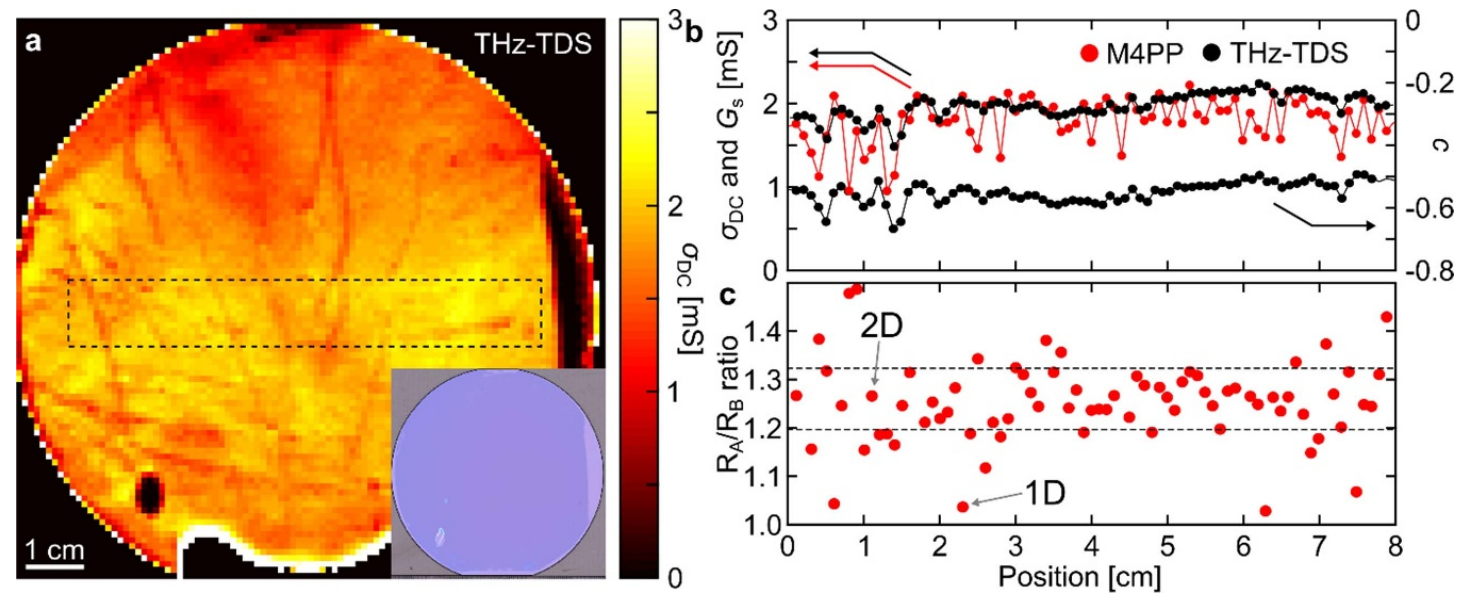

Figure 4. (a) THz-TDS map of $\sigma_{\mathrm{DC}}$ for graphene transferred to an oxidized 4 inch HR-Si wafer. Inset shows optical map of the sample generated by stitching together multiple, high-resolution optical images. (b) Line plot of $\sigma_{\mathrm{DC}}$ taken inside the rectangle in (a) together with M4PP $G_{\mathrm{s}}$ values from a scan along the same line. The THz-TDS Drude-Smith $c$ parameter along the same line is also shown on the opposite $y$-axis. (c) Line plot of the $R_{\mathrm{A}} / R_{\mathrm{B}}$ resistance ratio for the M4PP line scan shown in (b). The dashed lines indicate $\pm 5 \%$ deviation from a $R_{\mathrm{A}} / R_{\mathrm{B}}$ resistance ratio of 1.26 .

spectra are non Drude-like. The dips in conductivity are clearly more substantial for the M4PP measurement compared to THz-TDS. This discrepancy stems from an important difference between the two methods that becomes particularly apparent for non-continuous samples, i.e. with line-defects and tears. An electrical measurement, even with such small probe spacing as the M4PP $(10 \mu \mathrm{m})$, can be completely disrupted by a line defect between the source and drain. The THz-TDS measurement, however, measures an average (over the $\mathrm{THz}$ spot size of $\sim 400 \mu \mathrm{m}$ ) of the local conductivity in every spot of the graphene film, irrespective of whether the current can flow across a line defect or not. The characteristic length scale of this 'locality' is roughly given by the diffusion length, which is typically less than $100 \mathrm{~nm}$ [14]. It is also seen in figure $4(\mathrm{~b})$ that $c$ and $\sigma_{\mathrm{DC}}$ are seen to be correlated, implying that preferential backscattering has a detrimental impact on DC conductivity.

By performing M4PP measurements in two configurations (A, B) it is possible to determine whether the current flow in the sample is qualitatively one- or two-dimensional (1D or 2D) from the $R_{\mathrm{A}} / R_{\mathrm{B}}$ resistance ratio, where $\mathrm{A}$ and $\mathrm{B}$ correspond to two complementary current-voltage configurations, i.e. $\mathrm{A}\left(I_{1,4}, V_{2,3}\right)$ and $\mathrm{B}\left(I_{1,3}, V_{2,4}\right)$, following the notation described in detail elsewhere [14]. If $R_{\mathrm{A}} / R_{\mathrm{B}}=1$ the current flow is said to be $1 \mathrm{D}$-like, while a uniform conductor will exhibit $R_{\mathrm{A}} / R_{\mathrm{B}} \approx 1.26$ [72-74]. Figure 4(c) shows a line plot of the $R_{\mathrm{A}} / R_{\mathrm{B}}$ resistance ratio across the same line as in figure $4(\mathrm{~b})$. Interestingly, the M4PP $R_{\mathrm{A}} / R_{\mathrm{B}}$ resistance ratio shows that the current flow is overall $2 \mathrm{D}$-like 
despite the Drude-Smith backscattering parameter $c$ of around -0.55 , which indicates that there is a reasonable degree of carrier localization. This highlights that further work towards understanding of the exact physical interpretation of non-Drude-like conductivity spectra in THz-TDS for graphene is necessary.

\subsection{Reference-free analysis}

For THz-TDS measurements of samples where the individual transients from the directly transmitted pulse and the internal reflections are wellseparated in time (see figure $1(\mathrm{~d})$ ) it is possible to extract $\tilde{\sigma}_{s}(\omega)$ without performing the standard reference scan [29]. This is desirable for substrates that are fully covered by graphene where the reference measurement has to be performed on a different sample and in production scenarios where a THz-TDS system will be running for extended periods with possible fluctuations from $\mathrm{THz}$ source, environment, and mechanical movement being very likely.

An optical map and the corresponding graphene coverage map of a sample is shown in figures 5(a) and (b). The graphene coverage map was generated by comparing the red, green, and blue contrast from each recorded pixel of the optical map to the theoretically calculated contrast for single layer graphene (SLG) and bilayer graphene on $90 \mathrm{~nm} \mathrm{SiO}_{2}$ substrate [75]. The graphene is homogeneously single layer and covers the sample continuously, except from some small holes at the center left. A higher magnification image (figure 5(c)) also confirms that the graphene is continuous on a smaller scale. Conductivity maps obtained from standard referenced analysis (equation (2)) and reference-free analysis (equation (4)) are shown in figures 5(d) and (e) and look very similar. Both are obtained by fitting each spatial point measurement with the Drude model. The conductivity looks homogeneous at $\sim 2.5 \mathrm{mS}$ for both maps with a small region of lower conductivity at the center left, where the optical images show a small hole. The histograms for $\sigma_{\mathrm{DC}}, \tau, \mathrm{n}$, and $\mu$ in figures 5(f)-(i) show very similar distributions, where the median values vary very little $(<5 \%)$, which is also the case for $\nu_{\mathrm{F}}^{*}$ as shown previously [29] and shows that the method is certainly applicable. The data shown here for reference-free analysis includes a Gouy phase shift correction, while the amplitude correction is not necessary for HR-Si with negligible absorption [76] at low $\mathrm{THz}$ frequencies. Similar results for median values are obtained by using the referencefree analysis without Gouy phase shift correction, which, however, results in a larger spread in the histograms.

The graphene sample used for referencefree analysis was grown to ensure a Drude like frequency-dependent conductivity. Adlayers and grain boundaries, as discussed in section 2.2, can degrade the performance of graphene, and result in inhomogeneous performances from device to device $[58,77,78]$. The grain boundaries occur when graphene grains of different crystallographic orientations join together during CVD growth of large area graphene on metallic (normally Cu) surfaces [58, 79], while adlayers (bilayer or multilayer regions nucleated from the subsurface carbon in a $\mathrm{Cu}$ substrate) are almost always present in CVD grown graphene films [77].

Epitaxial growth of graphene on a single crystal substrate (e.g. $\mathrm{Cu}(111), \mathrm{Cu} / \mathrm{Ni}(111)$ alloy surface) with a lattice spacing closely matched to graphene is promising to achieve single crystal graphene film over a large area. Aligned graphene islands have been reported to grow on the surface of single crystal $\mathrm{Cu}(111)$ and $\mathrm{Cu} / \mathrm{Ni}(111)$ alloy and to merge without the presence of grain boundaries in the joining regions [77, 80-84]. Note that commercial polycrystalline metal foils can be reproducibly converted to single crystal $\mathrm{Cu}(111)$ (and other metal foils including $\mathrm{Ni}(111), \operatorname{Pt}(111)$, and $\mathrm{Co}(0001))$ using a contact-free annealing method under $\mathrm{Ar}$ and $\mathrm{H}_{2}$, while single crystal $\mathrm{Cu} / \mathrm{Ni}(111)$ alloy foils with fine tuning of the $\mathrm{Ni}$ concentrations can be generated by electroplating various amounts of $\mathrm{Ni}$ on both sides of the produced $\mathrm{Cu}(111)$ foil followed by an extra annealing [80, 85, 86]. Annealing in hydrogen gas atmosphere is found to deplete any subsurface carbon in a $\mathrm{Cu}$ foil, thus adlayer-free single crystal graphene film can be grown on $\mathrm{Cu}(111)$ foils made by contact-free annealing [86]. The single crystal graphene film used here contains parallel, centimeterlong, $\sim 100 \mathrm{~nm}$ wide folds (formed due to the thermal contraction of the $\mathrm{Cu}(111)$ foil during cooling) separated by $20-50 \mu \mathrm{m}$. Though the carrier mobility of a graphene field-effect transistor is significantly decreased if the active region contains fold(s), the highly-oriented folds allow straightforward fabrication of devices in the regions between adjacent folds, because these regions are homogeneously single layer and single crystal [77]. The single crystal nature of the graphene film and the lack of adlayers limits the number of defects and grain boundaries that typically lead to Drude-Smith like features in the conductivity spectrum.

\subsection{Encapsulated graphene}

In order to achieve consistent, high quality electronic performance of graphene devices it is necessary to encapsulate them to shield the graphene layer from the surrounding environment due to adsorbates [87, 88]. This encapsulation of graphene can be achieved by the use of dielectric 2D materials such as hexagonal boron nitride (hBN) [89] or atomic layer deposition (ALD) of dielectrics such as $\mathrm{Al}_{2} \mathrm{O}_{3}$ [90-92]. However, once graphene samples are encapsulated it is difficult to measure the electrical properties without specialized contact 

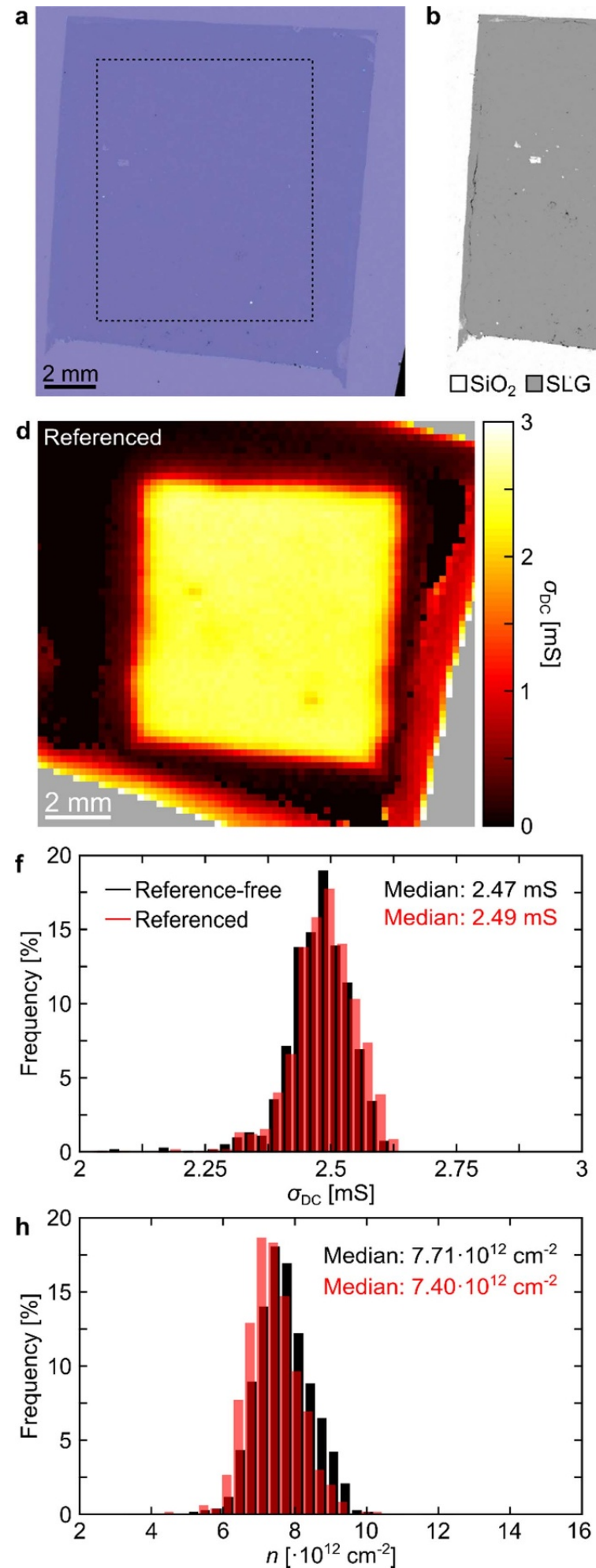

b

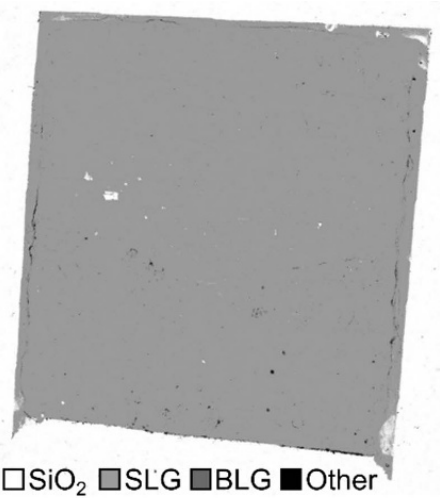

C
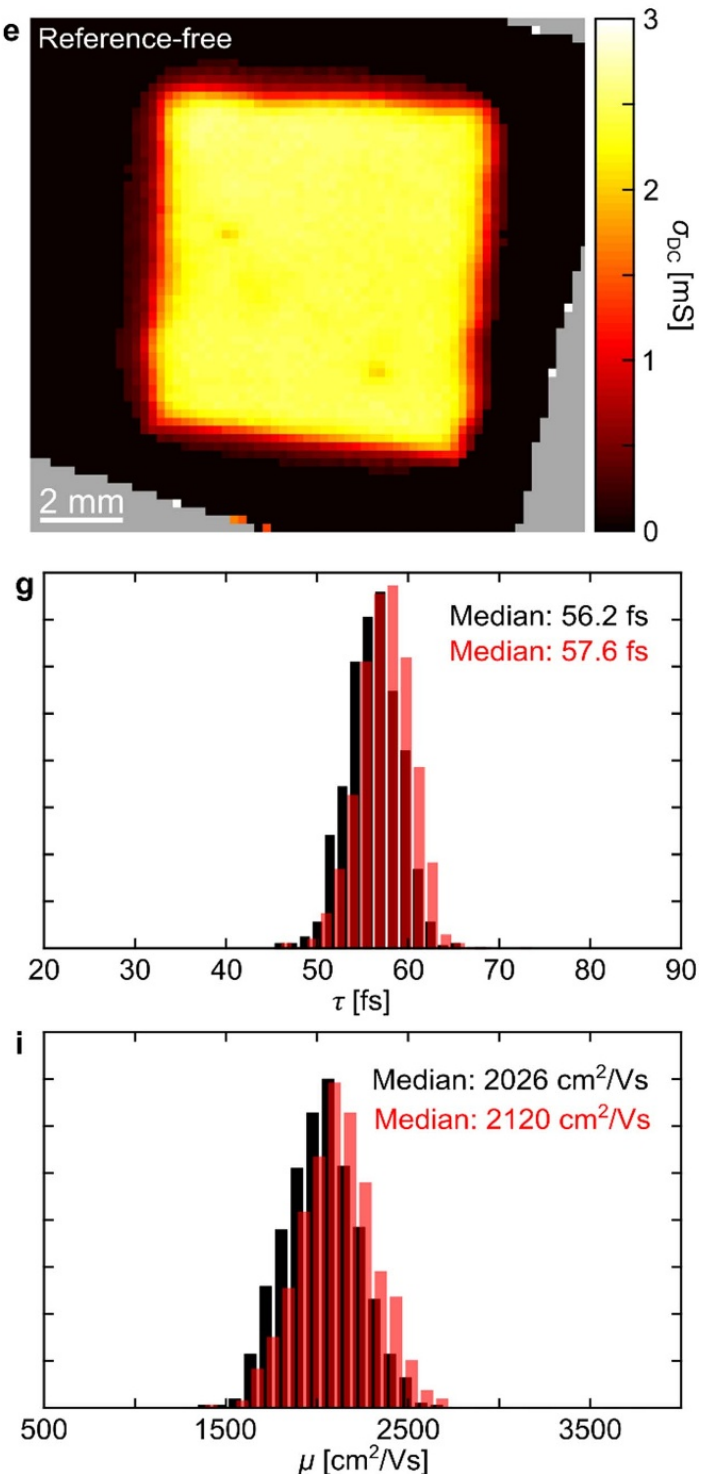

Figure 5. (a) Optical map of graphene on $90 \mathrm{~nm} \mathrm{SiO} / 2 / \mathrm{Si}$ substrate. (b) Graphene coverage map of the sample shown in (a). (c) High magnification image from sample shown in (a). (d), (e) THz-TDS $\sigma_{\text {DC }}$ maps of graphene sample shown in (a) based on (d) standard referenced analysis and (e) reference-free analysis. (f)-(i) Histograms with comparisons of electrical parameters extracted from reference-free and standard referenced analysis. (f) $\sigma_{\text {DC }}$ (g) $\tau$. (h) n. (i) $\mu$. Values for histograms are from within dotted square in (a).

designs and following complex sample fabrication [93]. This is inconvenient and expensive for the finished encapsulated material and following the impact of intermediate process steps on the electronic performance becomes nearly impossible.
Since the dielectric materials used for encapsulation are generally transparent to $\mathrm{THz}$ radiation, THz-TDS can be used to assess the electrical properties of encapsulated graphene, as well as after each of the process steps leading to the final 

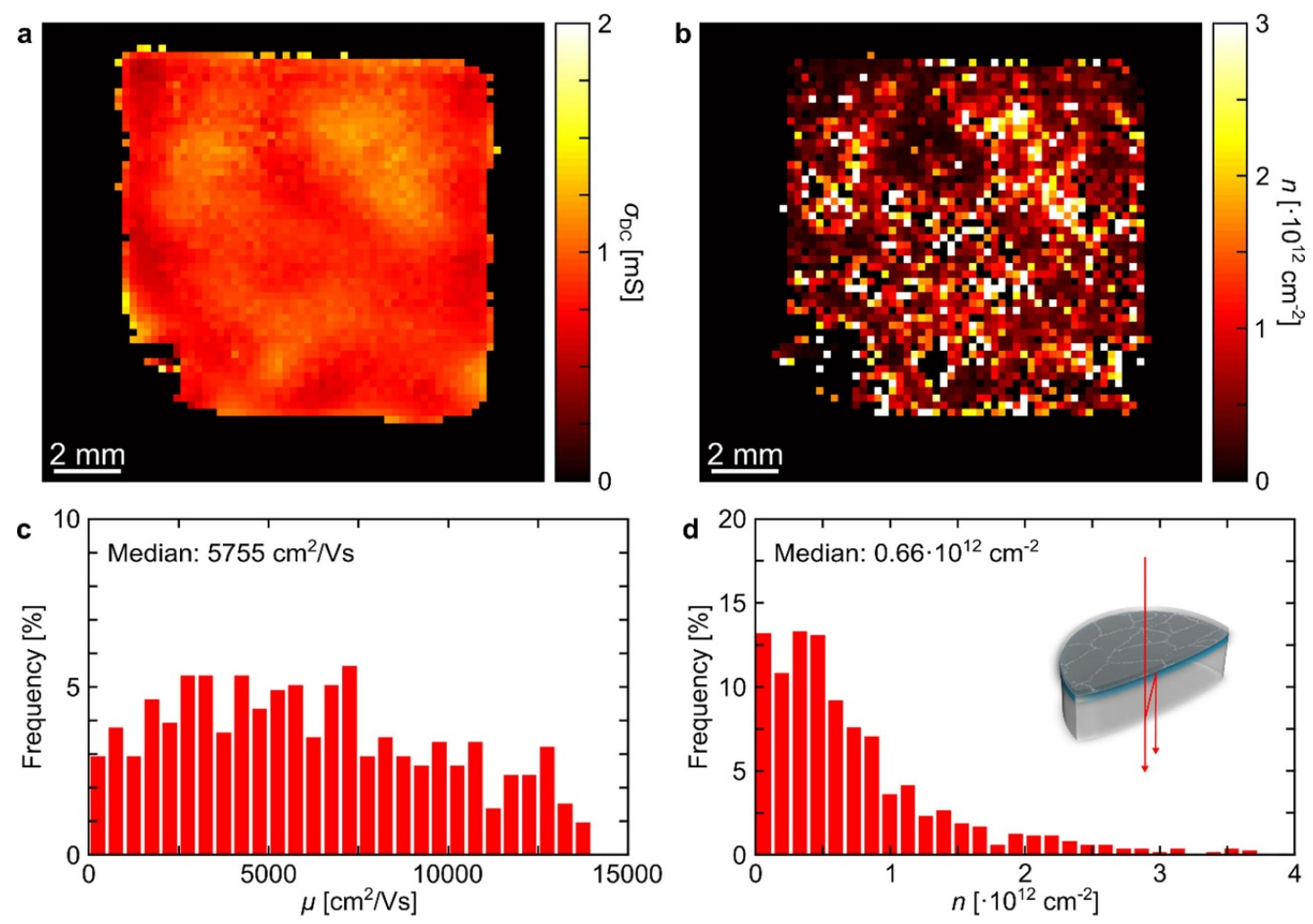

Figure 6. THz-TDS measurement of ALD encapsulated graphene. (a), (b) Maps of $\sigma_{\mathrm{DC}}$ and n. (c), (d) Histograms of $\mu$ and $n$.

results, simply because it is non-destructive and noninvasive. This has already been done for hBN encapsulation, where THz-TDS and Hall device measurements gave very similar results [46].

Here we show that the electrical properties can also be determined for graphene encapsulated by $30 \mathrm{~nm} \mathrm{ALD} \mathrm{Al}_{2} \mathrm{O}_{3}$ using a process described in detail by Mzali et al [92]. Since the $10 \times 10 \mathrm{~mm}^{2}$ sample is fully covered by encapsulated graphene, we use the reference-free analysis (equation (4)) without corrections to extract the electrical properties from the THzTDS measurement with all spectra fitted with the Drude model. Figure 6(a) shows a map of $\sigma_{\mathrm{DC}}$ for the sample. The conductivity is split into areas of higher and lower conductivity, which is even more distinct in the map of $n$ (figure 6(b)). From the THz-TDS measurement itself it is not possible to determine whether this originates from the graphene growth or transfer process. Although the spread is relatively larger, the histograms of $\mu$ and $n$ in figures 6(c) and (d) shows values that compare well to results from similarly fabricated graphene field effect transistors (FETs) [92], where mobility values of $\sim 6000 \mathrm{~cm}^{2} \mathrm{~V} \mathrm{~s}^{-1}$ are reported. An even better match appears for the distribution of $n$, which is very similar to the FET results [92], with a majority of the distribution $<0.5 \times 10^{12} \mathrm{~cm}^{-2}$ and a steady decrease that ends at $4 \times 10^{12} \mathrm{~cm}^{-2}$.

\subsection{THz-TDS for graphene quality control}

THz-TDS has so far mainly been used for graphene characterization after transfer due to growth most commonly being carried out on metallic surfaces, in particular $\mathrm{Cu}$. However, the clear advantage of direct growth on dielectrics, emphasizes the importance of being able to carry out characterization on as-grown graphene on such substrates. Here we show examples of how THz-TDS can be employed for quality control and process optimization in different steps of the fabrication cycle of graphene.

\subsubsection{Growth optimization}

$\mathrm{SiC}$ was one of the first $\mathrm{THz}$ transparent substrates that graphene growth was conducted directly onto $[34,94]$. Graphene has since also been grown on $\mathrm{SiN}$ [95], glass [96, 97], and sapphire [10]. In figure 7 we show examples of THz-TDS mapping of as-grown graphene on 4 inch sapphire wafers [10], which were produced using different graphene growth conditions. The result seen in figure $7(\mathrm{a})$ is symptomatic of a deposition onto a bowed wafer at high temperature; this can be resolved by precise control of the temperature ramping and heat distribution over the wafer. The sample in figure $7(\mathrm{~b})$ has incomplete graphene growth, which is resolved by optimizing the carbon source flow and exposure time. The conductivity maps in figures $7(\mathrm{a})$ and (b) obtained from fitting with the Drude-Smith model show clear inhomogeneity in $\sigma_{D C}$ across the wafer, while the conductivity achieved is much more homogeneous in figure $7(\mathrm{c})$ after tuning the process conditions. This is clearly seen in the histogram in figure $7(\mathrm{~d})$, where the standard deviation drops from $21.4 \%$ and 

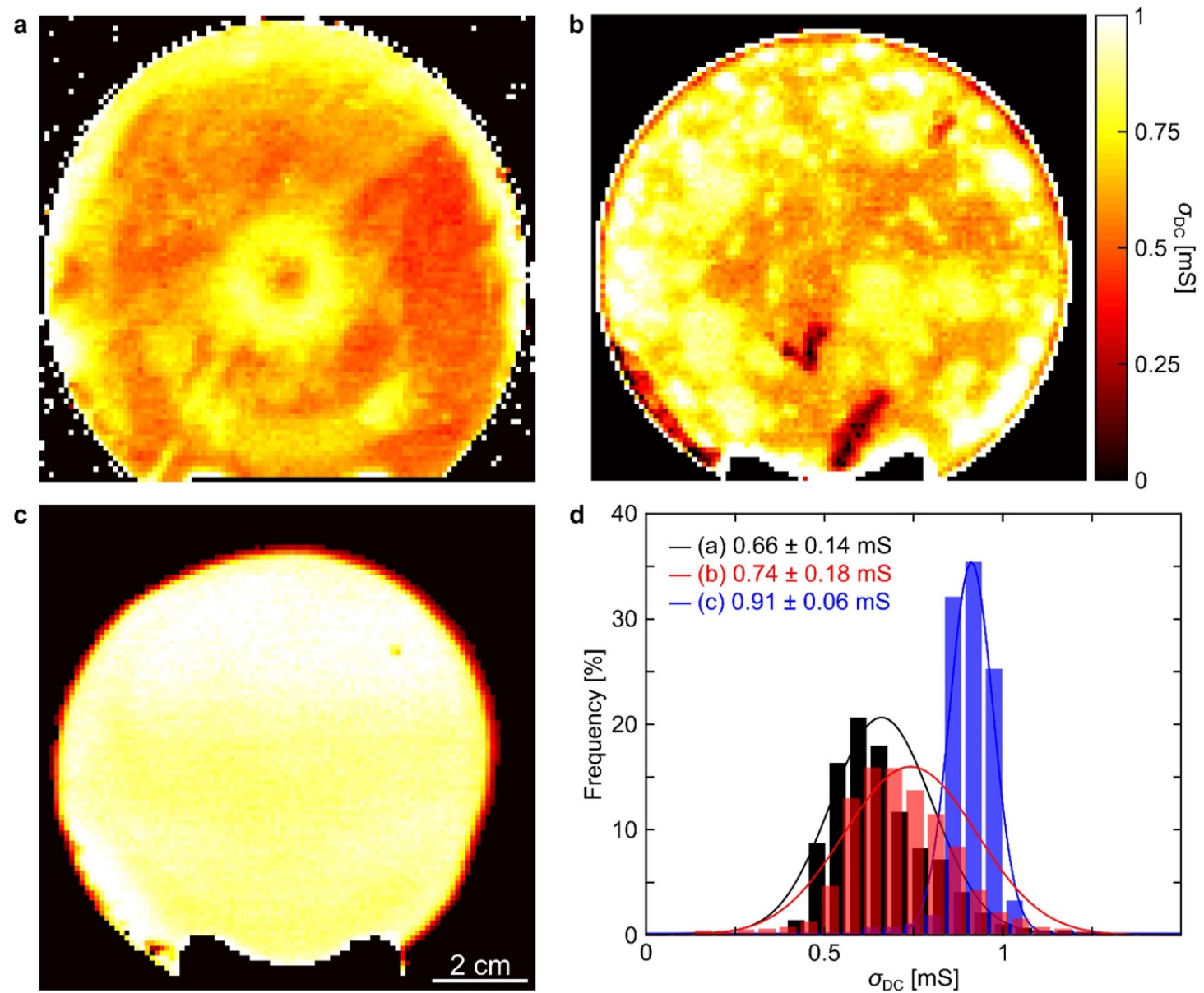

Figure 7. (a)-(c) THz-TDS conductivity maps of graphene as-grown on sapphire substrates using different growth conditions. (d) Histogram of $\sigma_{\mathrm{DC}}$ for the maps in (a)-(c). The scale bar and colorbar is the same for (a)-(c). (c) Adapted from [10].

$24.9 \%$ in (a) and (b) to only $6.6 \%$ for (c). By waferscale mapping of the electrical properties of as-grown samples before any transfer processing it becomes significantly easier to perform direct optimization of the graphene growth process without having to disentangle whether defects measured post-transfer are growth-related or transfer-related.

\subsubsection{Transfer optimization}

Intercalation of water between graphene and the growth substrate has led to an advancement in transfer methods that are non-destructive towards the graphene growth substrate [98-101]. Here we use THz-TDS to measure the conductivity of graphene grown on sapphire [10] before and after attempted water intercalation in order to investigate the effects of a similar process for this system. Figures $8(a)$ and (b) shows THz-TDS conductivity maps of graphene on sapphire before and after attempted waterintercalation (de-ionized water, $50^{\circ} \mathrm{C}, 24 \mathrm{~h}$ ). The $24 \mathrm{~h}$ submersion in water seemed to have little effect on the measured conductivity as also seen in figure 8(c), where the average conductivity for both maps is within the standard deviation from a fit of each histogram to a normal distribution.
Although there does not seem to be a measurable effect of the water submersion on the THz-TDS results, we still find that the method gives useful information when performing a peeling-based transfer using poly(vinyl alcohol) (PVA) as the transfer polymer [101]. Figures $8(\mathrm{~d})$ and (e) shows THzTDS conductivity maps of a sample of as-grown graphene on sapphire and the same graphene layer after PVA-based transfer to an oxidized HR-Si substrate. The sample seems to contain more holes after transfer as also observed in the graphene coverage map (figure 8(f)) and the conductivity is clearly lower after transfer compared to as-grown on sapphire (figure $8(\mathrm{~g})$ ). Just as THz-TDS can benefit growth characterization (section 3.4.1) it can also be used to determine whether defects in transferred graphene origin from the growth process or from the transfer process, and thereby aid in disentangling otherwise complex process diagnostics. Although the lower graphene conductivity after transfer may be due to the additional holes and defects in the graphene layer we cannot completely rule out that a difference in the coupling between graphene and substrate (sapphire vs $\mathrm{SiO}_{2}$ ) also has a role to play.

An example of how THz-TDS can be used to spot inhomogeneities in graphene on oxidized HR-Si 


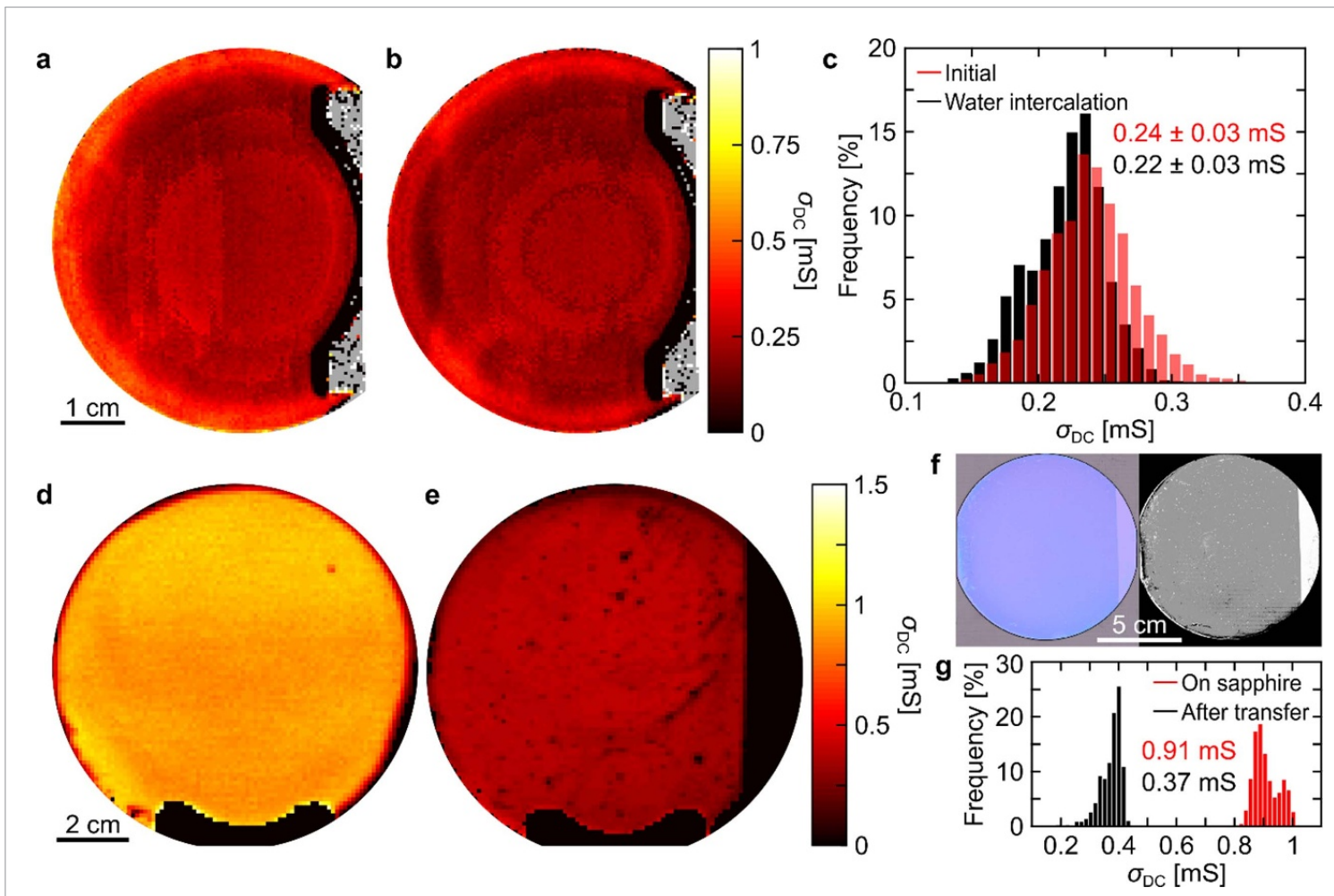

Figure 8. (a), (b) THz-TDS conductivity maps of graphene on 2 inch sapphire wafer (a) as-grown and (b) after attempted wafer-intercalation. (c) Histogram of $\sigma_{\mathrm{DC}}$ for the central region of the maps in (a), (b). (d), (e) THz-TDS conductivity maps of graphene (d) as-grown on 4 inch sapphire wafer and (e) after transfer to oxidized HR-Si substrate. (f) Optical and graphene coverage maps of the sample in (e). (g) Histogram of $\sigma_{\mathrm{DC}}$ for the central region of the maps in (d), (e). Panels (d), (f) and (g) are reprinted with permission from [10] Wiley.

that appears homogeneous from optical microscopy is shown in figures $9(a)$ and (b) that shows an optical and THz-TDS conductivity map of the same sample. From the optical map the sample appears homogeneous, except from a hole near the upper right corner. That hole is also visible in the THz-TDS conductivity map, which also highlights several additional features in the sample. The conductivity does not appear homogeneous but varies in a patchwork style. The sample was further measured by Raman spectroscopy to verify whether the conductivity variation measured by THz-TDS was real. Figure 9(c) shows a map of the Raman $\mathrm{G}$ peak position, which has been correlated to the doping level of the graphene layer $[102,103]$. The map of the Raman G peak exhibits the same features as the THz-TDS conductivity map and confirms that the pattern observed in the conductivity is in fact real and not a measurement artefact.

We speculate that the peculiar pattern in the conductivity shown in figure 9 (b) is caused by inhomogeneous application of pressure in a stamping system for graphene transfers. The correlation between the THz-TDS conductivity and Raman G peak has also been discussed previously [41], and THz-TDS has earlier proven to be able to highlight defects in graphene on PET not visible to the eye, or even with optical microscopy [11].

\subsubsection{Post-transfer optimization}

THz-TDS can be used to measure the electrical properties of encapsulated graphene as shown in section 3.3 for $\mathrm{ALD} \mathrm{Al}_{2} \mathrm{O}_{3}$ passivated graphene. This demonstrates that THz-TDS can be used to optimize such layers by performing fast, non-destructive measurements of the electrical properties between process steps. $\mathrm{Al}_{2} \mathrm{O}_{3}$ encapsulation of graphene can be performed in a two-step process (figure 10(a)), where a thin $(1 \mathrm{~nm}) \mathrm{AlO}_{x}$ protection layer is first deposited by electron beam evaporation followed by an ALD $\mathrm{Al}_{2} \mathrm{O}_{3}$ passivation layer [92]. Here we perform several $\mathrm{THz}$-TDS measurements of four samples to reveal the influence of each process step; first we measured the bare graphene layer, then we measured again after $\mathrm{AlO}_{x}$ protection was added, and finally after full ALD $\mathrm{Al}_{2} \mathrm{O}_{3}$ passivation. The measurement time per sample was $<10 \mathrm{~min}$ for each step.

Figures 10(b)-(f) show bar plots of the evolution in $\sigma_{\mathrm{DC}}, \tau, \mathrm{n}, \mu$, and $\nu_{\mathrm{F}}^{*}$ for each process step for the four samples. There was no ALD $\mathrm{Al}_{2} \mathrm{O}_{3}$ passivation layer added for sample 1 for reference purposes. While the initial conductivity is relatively similar for all four samples (within $~ 5 \%$ ), there is more variation for $\tau, \mathrm{n}$, and $\mu$. The general trends are decrease and stabilization of $\sigma_{\mathrm{DC}}$ after $\mathrm{AlO}_{x}$ protection, with little further decrease after $\mathrm{ALD} \mathrm{Al}_{2} \mathrm{O}_{3}$ passivation. After both process steps $\tau$ and $\mu$ increases 
a

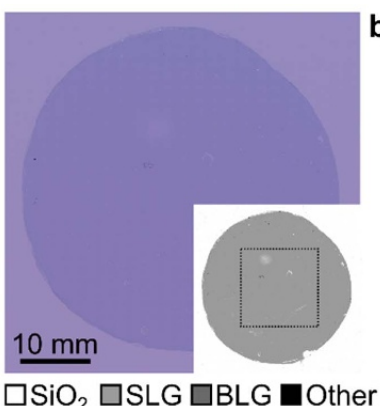

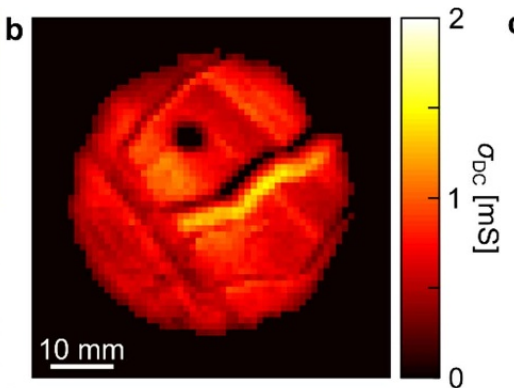

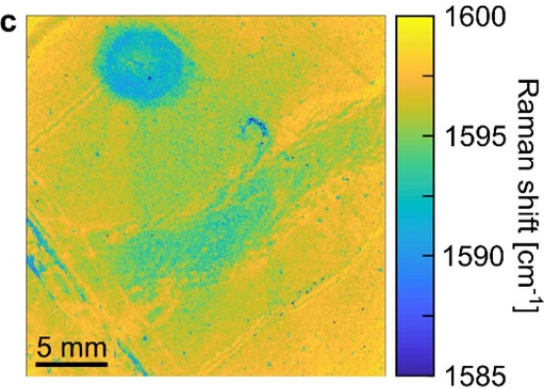

1585

Figure 9. (a) Optical map of 2 inch graphene area transferred from $\mathrm{Cu}$ growth substrate to oxidized HR-Si. Inset shows the corresponding graphene coverage map. (b) THz-TDS map of $\sigma_{\mathrm{DC}}$ for the sample in (a). (c) Map of the Raman G-peak position from the region highlighted by dotted square in the graphene coverage map in (a).

a

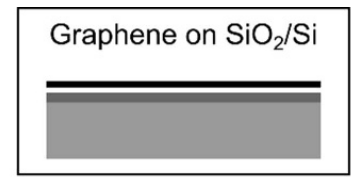

b

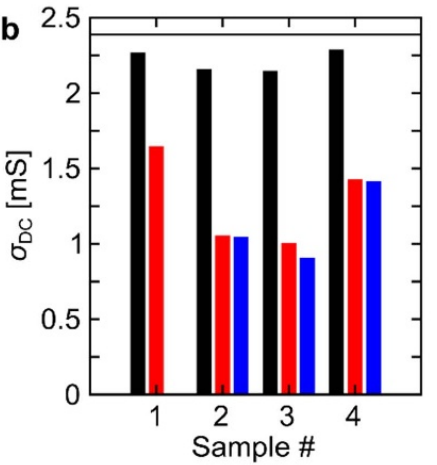

e

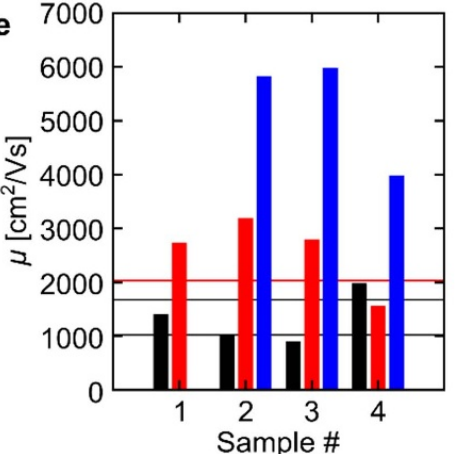

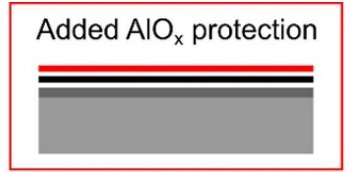
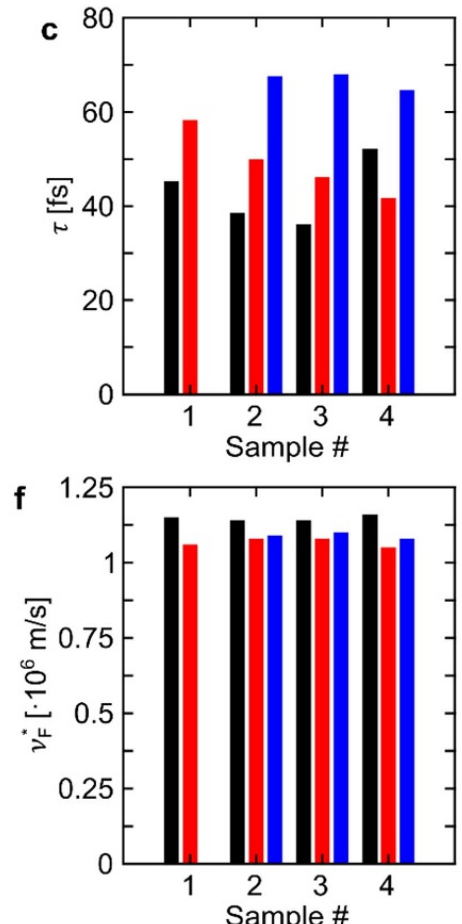
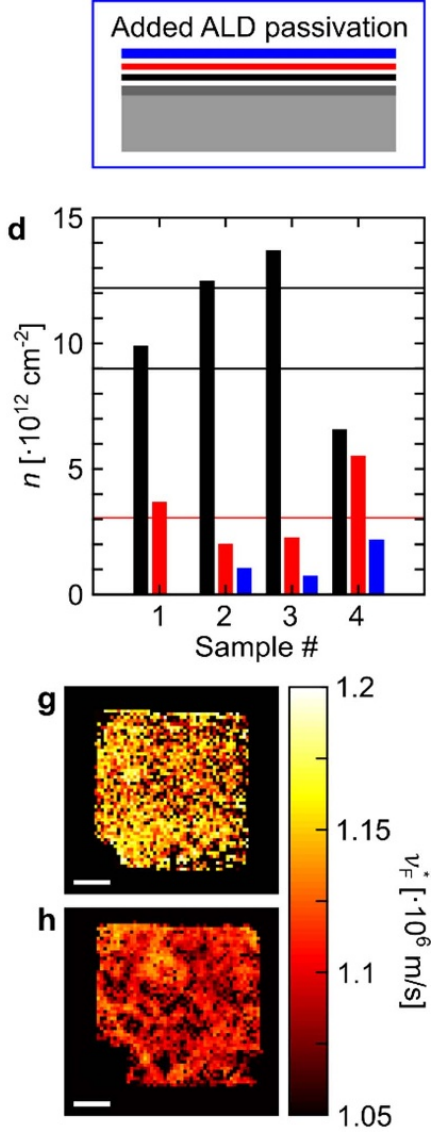

Figure 10. (a) Illustration of process flows for passivation of graphene, showing a sample without passivation (black box), with $\mathrm{AlO}_{x}$ protection (red box), and with protection and $\mathrm{ALD} \mathrm{Al} \mathrm{O}_{3}$ passivation (blue box). (b)-(f) Bar plots of the evolution in electrical properties (median value for $\sigma_{\mathrm{DC}}, \tau, \mathrm{n}, \mu, \nu_{\mathrm{F}}^{*}$ ) of graphene for each process step for four different samples. The process step is indicated by the color of the bars, which correspond to the color of the boxes in panel (a). Horizontal lines in (b), (d), (e) indicate values measured by complementary technique on the same samples with process step indicated by color of the line. (g), (h) THz-TDS maps of $\nu_{\mathrm{F}}^{*}$ for sample 3 (g) without passivation and (h) after protection and passivation processes.

while $n$ decreases. For $\nu_{\mathrm{F}}^{*}$ there is a decrease after $\mathrm{AlO}_{x}$ protection and a small increase after $\mathrm{ALD} \mathrm{Al}_{2} \mathrm{O}_{3}$ passivation as also seen in the $\nu_{\mathrm{F}}^{*}$ maps in figures $10(\mathrm{~g})$ and $(\mathrm{h})$. The change in $\nu_{\mathrm{F}}^{*}$ from bare to processed graphene is mainly driven by the fact that we use a value of 9.4 for the relative permittivity of the deposited aluminum oxide [104] instead of 1 for air in equation (8).
Several reference measurements were conducted on the same samples to validate the THz-TDS results and the change of relative permittivity for aluminum oxide. These measurements are indicated by horizontal lines in figures 10(b), (d) and (e). A sheet conductance of $\sim 2.4 \mathrm{mS}$ for initial samples was measured by a Lehighton LEI 1500E, which matches the THz-TDS $\sigma_{\mathrm{DC}}$ values within $10 \%$. Hall 

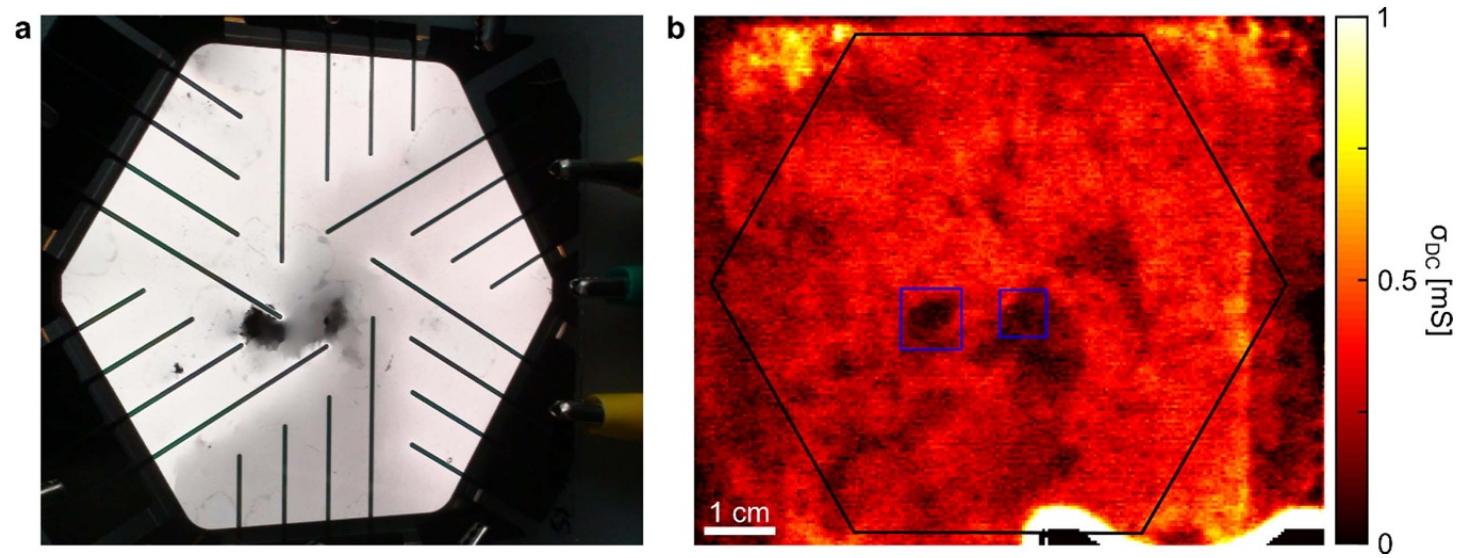

Figure 11. (a) Photograph of light emission from a white OLED with graphene as transparent electrode (anode). Image is courtesy of Fraunhofer FEP. (b) THz-TDS conductivity map of the graphene on quartz sample used for OLED in (a) before deposition of active layers and metallic back side contact. Blue squares in (b) highlight the position of the two clearly visible defects in the OLED in (a).

effect measurements of $n_{\mathrm{H}}$ and $\mu_{\mathrm{H}}$ were conducted for samples before and after $\mathrm{AlO}_{x}$ protection, with reasonable agreement with the $\mathrm{THz}$-TDS values. No measurements were performed on these samples after ALD $\mathrm{Al}_{2} \mathrm{O}_{3}$ passivation due to difficulties in fabrication of side contacts. However, for samples fabricated similarly to sample 3 , it was previously shown [92] that $n$ is generally below $3 \times 10^{12} \mathrm{~cm}^{-2}$ after $\mathrm{AlO}_{x}$ protection and below $1 \times 10^{12} \mathrm{~cm}^{-2}$ after ALD $\mathrm{Al}_{2} \mathrm{O}_{3}$ passivation, while $\mu$ can reach values of $\sim 6000$ $\mathrm{cm}^{2} \mathrm{~V} \mathrm{~s}^{-1}$ after $\mathrm{ALD} \mathrm{Al}_{2} \mathrm{O}_{3}$ passivation. These results all agree well with the THz-TDS values reported here.

We note that different deposition parameters and $\mathrm{Al}_{2} \mathrm{O}_{3}$ thicknesses (15-30 nm) were used for the four samples and the initial values for the electrical properties varied between samples, so exactly similar results for the four samples should not be expectedindeed, these results show how THz-TDS can be used for optimizing process parameters of the ALD process flow. THz-TDS could also work for layers buried deeper inside the substrate. This, however, would require changes to the transmission function model used for parameter extraction from the system.

Another example of the use of THz-TDS in quality control is for early diagnosis and testing prior to device finalization in relation to transparent electrode applications, where the graphene layer will usually be present on a transparent substrate. One example is organic light emitting diodes (OLEDs) where graphene can be transferred to a glass or polymeric substrate before deposition of active organic layers and a (often metallic) back side contact. THzTDS can be used to screen the quality (conductivity, homogeneity) of the graphene layer before initiating further process steps. An example of how this can benefit is shown with a white graphene-based OLED in figure 11 (a). The active area is $42.3 \mathrm{~cm}^{2}$ and the OLED emits relatively uniformly, except from two clear defected areas close to the center of the sample.
These two defects were also present in a THz-TDS conductivity map of the graphene on quartz substrate before OLED processing (figure 11(b)), while not visible to optical inspection. Previous results have also shown that THz-TDS is effective for measuring the effects of chemical post-transfer doping of graphene to increase the conductivity [14] and for comparing electrical properties of graphene before and after device fabrication $[105,106]$.

\subsection{THz-TDS in production scenarios}

In section 3.4 we showed several examples of how THz-TDS can be used to optimize both growth, transfer, and post-transfer processing of graphene. Since the production of graphene has matured to a point, where large samples of graphene on 12 inch $\mathrm{Si}$ [107] and $25 \times 30 \mathrm{~cm}^{2}$ PET [11] can be fabricated, we will discuss here how THz-TDS can be applied in real production scenarios in particular with respect to measurement speed and on relevant substrates.

\subsubsection{THz-TDS scanning of 12 inch wafers in $1 \mathrm{~h}$}

One method of fabricating large areas of graphene is batch fabrication, where graphene is grown simultaneously on a batch of growth substrates and with individual transfers to a target substrate. Since silicon is a standard substrate for most electronic devices, we here discuss how to rapidly characterize graphene on 12 inch HR-Si wafers. The exact time for a measurement scan to be rapid can be discussed, but here we show that it is possible to measure the conductivity of graphene across a 12 inch HR-Si wafer with $1 \mathrm{~mm}$ step size within $1 \mathrm{~h}$. Given the time and step size, this takes $0.04 \mathrm{~s}$ of measurement time per measurement point considering a square sample of $300 \times 300 \mathrm{~mm}^{2}$. The used commercial spectrometer (see section 2) acquires THz-TDS spectra at $100 \mathrm{~Hz}$. It is therefore maximally possible to measure and average four waveforms per measurement point for a $1 \mathrm{~h}$ scan. 
Such a scan was performed for the THz-TDS conductivity map shown in figure 12 (a). It is noted that the 12 inch diameter HR-Si wafer is only covered by 4 inch diameter graphene placed centrally on the wafer due to fabrication constraints. However, the measurements were conducted in such a way that it would have taken exactly the same time and have the same precision if more or all of the wafer was covered by graphene as the full 12 inch wafer was mapped with the required scanning speed.

A reference measurement was performed in order to validate the THz-TDS conductivity map obtained from a $1 \mathrm{~h}$ scan of a 12 inch wafer. Several comparisons between $\sigma_{\mathrm{DC}}$ from THz-TDS and $G_{\mathrm{s}}$ from M4PP or Hall measurements have been made showing that THz-TDS works as an efficient probe of $\sigma_{\mathrm{DC}}$ for graphene $[5,6,8,9,11,14]$. The THz-TDS performed for such comparisons average over 20-50 waveforms per measurement point. The reference measurement was following performed with acquisition of 50 waveforms per measurement point and took $12.5 \mathrm{~h}$ to complete. The THz-TDS conductivity map of the reference measurement is shown in figure 12(b). The $1 \mathrm{~h}$ scan and the $12.5 \mathrm{~h}$ scan are nearly identical, despite a small shift in average conductivity, which is easily within the typical variations due to random fluctuations in humidity and temperature $[42,88]$. This is confirmed by the histogram of $\sigma_{\mathrm{DC}}$ from the two maps (figure 12(c)) that shows a slightly larger conductivity for the reference measurement. However, the deviation is only $4.1 \%$ when comparing average values from fitted normal distributions, which shows that is possible to perform trustworthy measurements of the conductivity of graphene with $1 \mathrm{~mm}$ step size across 12 -inch wafers within $1 \mathrm{~h}$.

We emphasize that the results for the $1 \mathrm{~h}$ scan of 12 inch wafers were obtained with a commercial setup that was introduced in 2007. Since then, progress in THz technology allows performance well beyond what is shown in figure 12, both in terms of speed and measurement accuracy. As an example, we show in figure 13 the frequency-dependent conductivity of graphene on $\mathrm{SiC}$ measured with the same setup used for figure 12 (and most other results presented in this work) compared to a Toptica TeraFlash Smart spectrometer based on electronically controlled optical sampling $[108,109]$. We note that the THz-TDS measurements for figure 13 were conducted on three different samples and that a comparison of the Drude model fits is not the aim of the figure. The improved frequency bandwidth with the new system is clearly seen, which should provide improved accuracy when fitting data with the Drude model. Furthermore, the new spectrometer is capable of acquiring THz-TDS spectra at $1600 \mathrm{~Hz}-\mathrm{a}$ $16 \times$ improvement compared to the setup used for figure 12. Considering the vast improvements in speed and spectral bandwidth offered by modern, yet affordable $\mathrm{THz}$ spectrometers in general we anticipate that 12 inch graphene wafer scans within $5-10 \mathrm{~min}$ is possible.

\subsubsection{Graphene on polymers for $R 2 R$ setups}

$\mathrm{R} 2 \mathrm{R}$ production of graphene is perhaps the most obvious method for large-area production, especially for graphene onto polymeric substrates, where graphene is grown on a rolling metallic foil and transferred onto a roll of polymeric material. Examples of graphene growth and transfer methods compatible with $\mathrm{R} 2 \mathrm{R}$ production have been shown already $[101,110,111]$. There are at present no options for inline characterization of the electrical properties of graphene in a $\mathrm{R} 2 \mathrm{R}$ production line, perhaps with the exception of THz-TDS. THz-TDS has proven to be capable of reliably measuring $\sigma_{D C}$ of graphene on PET substrate across $25 \times 30 \mathrm{~cm}^{2}$ samples (figure 14(a)) [11] and recently values of $n$ and $\mu$ measured for graphene on PET and polyethylene naphthalate (PEN) by THz-TDS were verified by reference measurements [12].

Figure 14(b) shows maps of $n$ and $\mu$ for graphene across $25 \times 30 \mathrm{~cm}^{2}$ substrates with corresponding histograms in figures 14(c) and (d). The maps of $n$ and $\mu$ appear homogeneous (standard deviation $<10 \%$ of average) in half of the map $(\sim 17500$ measurement points), but are inhomogeneous in the other half and that there is a clear vertical line pattern in the inhomogeneities. We ascribe these errors to drift in either the $\mathrm{THz}$ source or the mechanical stage and not the sample itself. This underpins a consideration that must be taken into account for R2R integration of THz-TDS systems. In this case the measurement artifacts could have been mitigated by using a THz-TDS system with short $\mathrm{THz}$ pulse duration making it possible to measure individual transients on thin polymer substrates (see figure 1) combined with reference-free analysis (section 3.2).

In figure 15 we show a THz-TDS conductivity map of a $26 \times 42 \mathrm{~cm}^{2}$ sample of graphene on PEN substrate. The sample is made by transferring individual $10 \times 10 \mathrm{~cm}^{2}$ samples into an array with overlapping regions as clearly seen in figure 15(a). This arrangement leads to patches of SLG with bands of two-layer and squares of four-layer graphene at the intersections. The conductivity clearly increases with increasing number of graphene layers as also shown in the bar plot in figure 15(b). The conductivity map of the graphene on PEN sample reveals many scratches and defects. As it turns out, all the scratches reside in the PEN substrate on the opposite side of the graphene - since they are relatively large and deep, they scatter the $\mathrm{THz}$ radiation before reaching the $\mathrm{THz}$ detector for those measurement points, which interferes with the determination of the graphene conductivity. Therefore, the quality and handling of the substrate must also be taken into consideration when designing a THz-TDS 


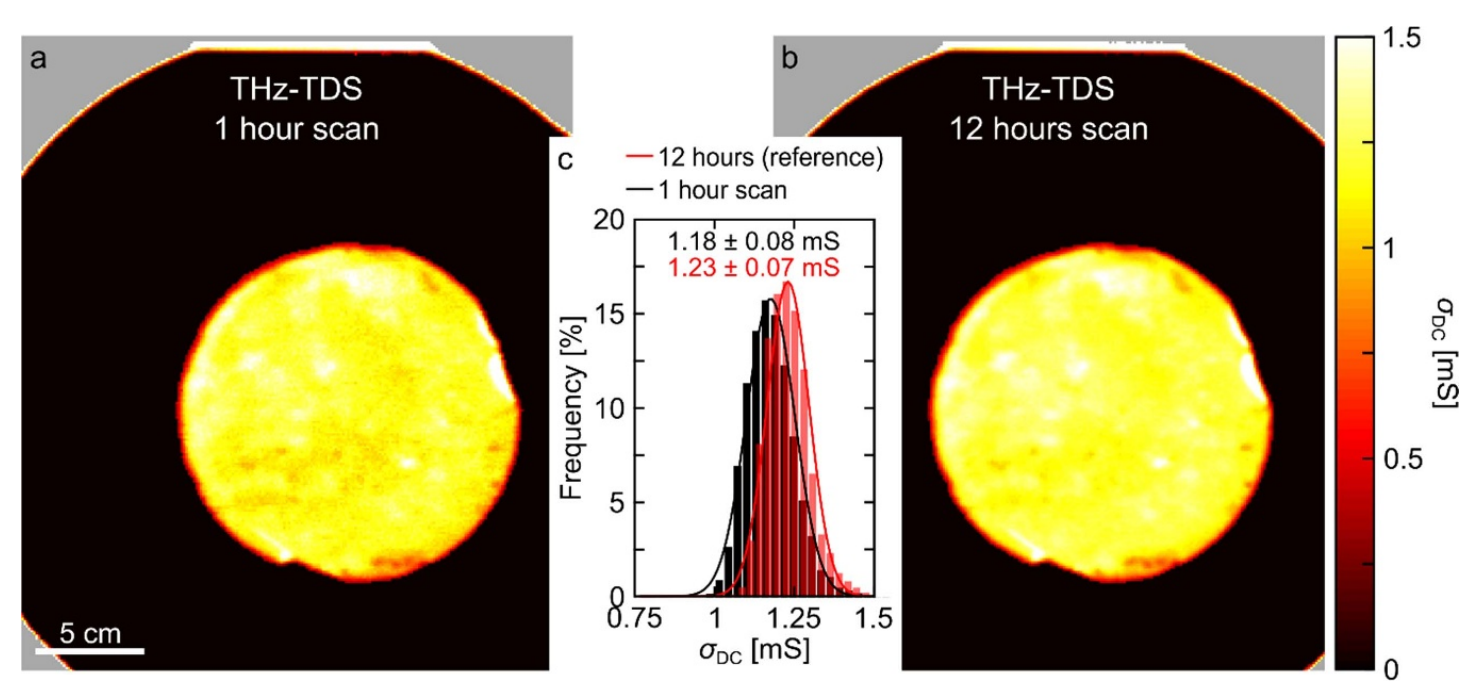

Figure 12. (a), (b) THz-TDS conductivity maps of graphene on 12 inch HR-Si wafer. The maps show results from measuring the same sample in (a) $1 \mathrm{~h}$ and (b) $12 \mathrm{~h}$. (c) Histogram of $\sigma_{\mathrm{DC}}$ for the central region of both maps.

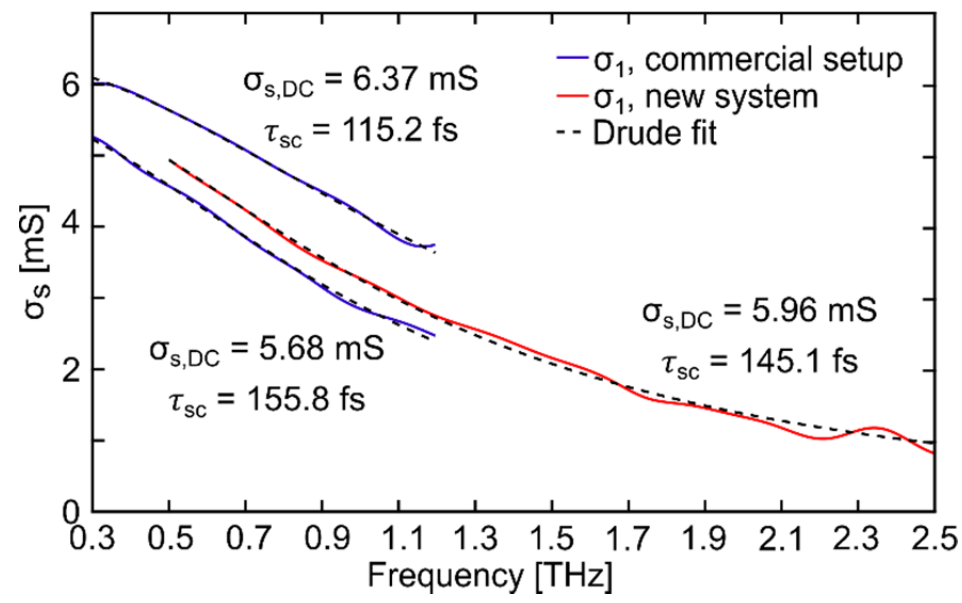

Figure 13. Comparison of THz-TDS frequency-dependent conductivity of graphene on SiC measured with the commercial setup used for most results presented in this work and a newer commercial setup with larger bandwidth and higher data acquisition rate. Reprinted with permission from [9] Elsevier.

setup for graphene production scenarios. Nevertheless, THz-TDS is uniquely capable of detecting defects in graphene not immediately visible for optical inspection [11].

The adaption of $\mathrm{THz}-\mathrm{TDS}$ into a $\mathrm{R} 2 \mathrm{R}$ production line could be based on a $\mathrm{THz}$ transmitter and receiver mechanically scanning back and forth across the graphene roll, perpendicular to the roll direction, but a more ideal solution would probably be to base it on fixed $\mathrm{THz}$ imaging scanners if proper resolution limits can be reached.

\subsubsection{A new IEC metrology standard}

As described above and in many other studies, the $\mathrm{THz}$-TDS technique is useful for conductivity mapping of graphene and other conductive thin films. However, for a more accepted use for quality control and documentation in the industry, it is imperative that a broadly accepted measurement protocol is established. Only by standardization will it be possible to compare results from different laboratories and production environments, and thereby to unfold the full potential of the technique. Based on the extensive volume of THz-TDS-based conductivity mapping of graphene that is now available in the literature, we and other partners in the EU Graphene Flagship have therefore developed a new international metrology standard for measurement of the conductivity of graphene under the auspices of the International Electrotechnical Commission (IEC). The standard, IEC TS 62607-6-10, has passed the approval phase of the EU Graphene Flagship and is expected to be published by the IEC in 2021. 


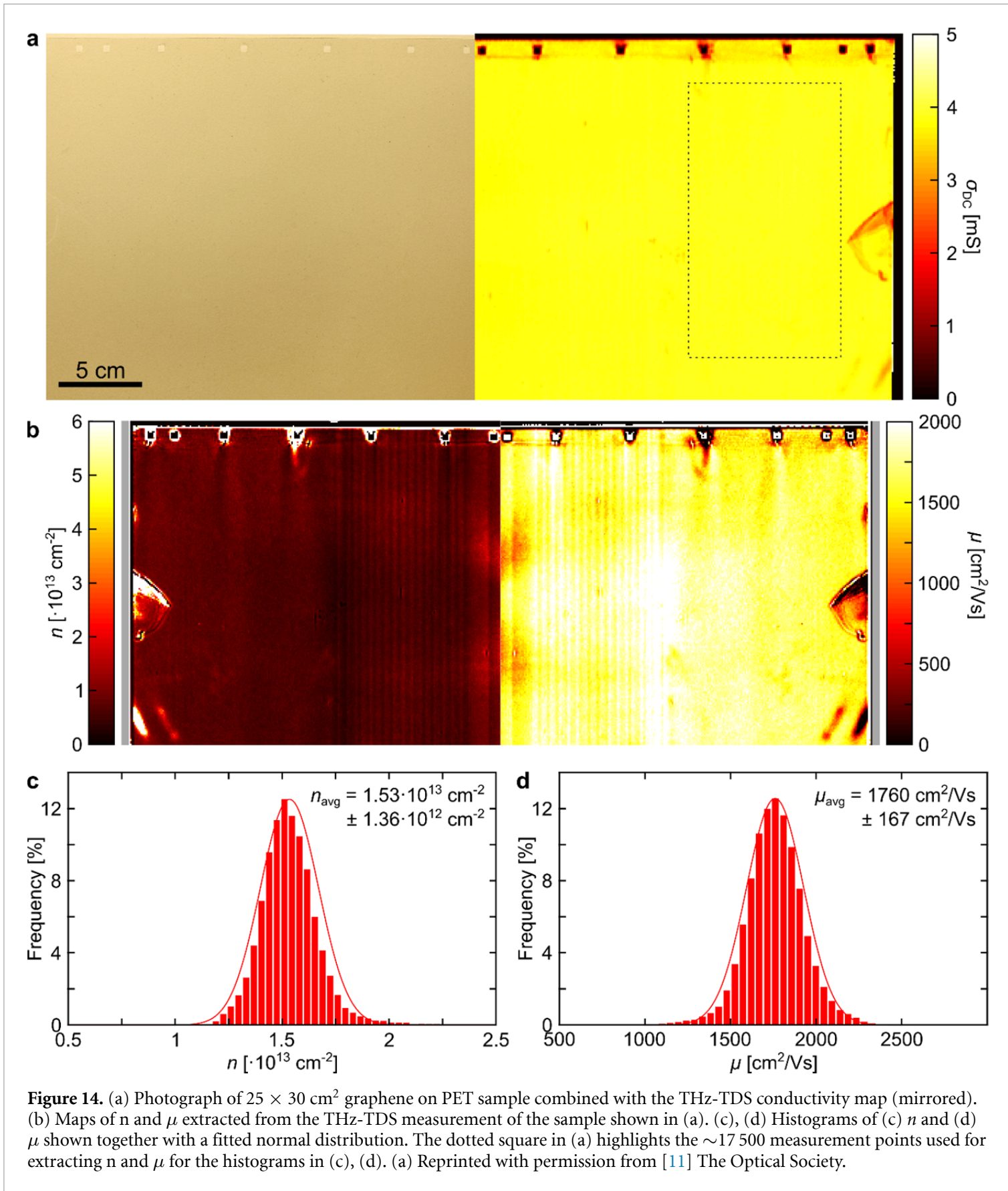

\section{Discussion}

We have provided an overview of different cases where THz-TDS proves useful for characterizing the electrical properties of graphene across a wide range of substrates, as well as different types and qualities of graphene. THz-TDS can provide large-area spatial maps of electrical key performance indicators for graphene which we find to be relevant in graphene production scenarios for both batch and $\mathrm{R} 2 \mathrm{R}$ production.

There are still obstacles to overcome for integration of THz-TDS in graphene pilot or production lines compared to a well-controlled laboratory environment where instrument down-time, measurement variabilities and artefacts can be dealt with effectively, as throughput is not an issue, and the experimental setup is located in an electrically and mechanically quiet environment. Such obstacles include long term stability of the THz-TDS metrology system and the reproducibility of measurements linked to this. In the case studies presented within this review, we find an overall good reproducibility of results when re-measuring samples over a short time scale (days), while over longer time scales the electrical properties measured from graphene changes due to environmental conditions. In a real-world production line, one would also have to address vibrational disturbances and mechanical drift that could lead to phase errors. Such vibrations could originate from other processes in the production environment that cannot be eliminated easily through mechanical 

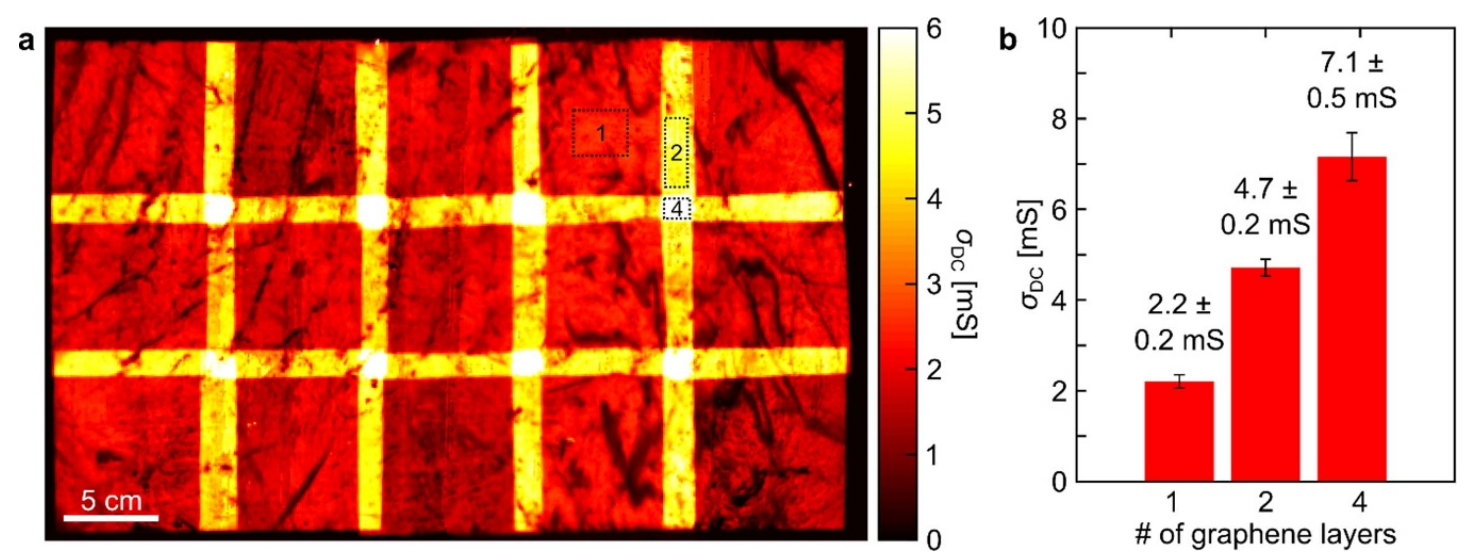

Figure 15. (a) THz-TDS conductivity map of tiled graphene samples on PEN. The total sample area is $26 \times 42 \mathrm{~cm}^{2}$. (b) Bar plot of the $\sigma_{\mathrm{DC}}$ values (average \pm standard deviation from fit to normal distribution) from the measurement pixels within the rectangles highlighted on map in (a).

insulation, or for instance from mechanical rollers in a R2R setup where the THz-TDS instrument itself is mounted. Another challenge is reflection mode measurements; these are highly desirable due to the lower price and simpler construction, better performance on non-transparent (terahertz absorbing) substrates and thereby greater utility and convenience. Reflection-based measurements are far more sensitive to variations in probe-sample distance than transmission measurements, and inherently more inaccurate for determination of carrier density and mobility of graphene. There are viable commercial solutions on the market now for inspection of graphene and other thin films (i.e. Das-Nano), but more work needs to be done to bring up reflection-based measurements to the same high standard as transmission measurements. Variations in probe-sample distance are also more critical when moving from far-field transmission mode (as in this paper) towards nearfield measurements, which improves the spatial resolution from hundreds of $\mu \mathrm{m}$ to few $\mu \mathrm{m}$ [46]. Commercial near-field $\mathrm{THz}$-TDS setups are also readily available from for instance Protemics $\mathrm{GmbH}$, which offer down to $10-50 \mu \mathrm{m}$ resolution on continuous graphene samples. The development of scatteringtype scanning near-field microscopy (s-SNOM, e.g. from Neaspec) setups operating at $\mathrm{THz}$ frequencies that can bring the spatial resolution down to tens of $\mathrm{nm}$ is also expected to contribute to the understanding of non-Drude $\mathrm{THz}$ spectra in the future. While primarily being research tools due to their slow speed and greater complexity of operation, such instruments could be highly valuable for R\&D and diagnostics.

The occurrence of non-Drude like features in the frequency dependent conductivity spectrum for THz-TDS measurements of graphene must also be further investigated. Here, we have used the phenomenological Drude-Smith model (see section 2.2) to determine $\sigma_{\mathrm{DC}}$, which yields results that compares well to M4PP measurements in section 3.1. There is, however, a lacking fundamental understanding of the link between a measured non-Drude spectrum and the physical appearance of the graphene sample (defects, grain boundaries, local contaminants, etc) and a variety of different models are being developed in the field [15, 66-70]. The use of Monte Carlo simulations is a possible route to reconcile the measurements with an analytical understanding, which is subject to further work.

By addressing these obstacles, the road to integration of THz-TDS into graphene production setups will be cleared, allowing contactless, accurate measurements of the key electrical properties for optimization of graphene growth, transfer, and post-transfer processes.

\section{Acknowledgments}

This work was partially funded by the European Union Graphene Flagship under Grant Agreement Nos. 785219 (Core 2) and 881603 (Core 3); Danish National Research Foundation Center for Nanostructured Graphene (DNRF103); European Union Horizon 2020 Research and Innovation Programme under Grant Agreement No. 824962 (car2TERA); European Union Seventh Framework Programme (FP7) under grant Agreement No. 604000 (GLADIATOR); Institute for Basic Science (IBS-R019-D1); National Nature Science Foundation of China (NSFC, Grants Nos. 51902306); Natural Science Pioneer Science Foundation of Chongqing of China (cstc2019jcyjxfkxX0006).

We acknowledge help with OLED fabrication from Beatrice Beyer and David Wynands (Fraunhofer FEP).

\section{ORCID iDs}

Patrick R Whelan @ https://orcid.org/0000-00023978-7029 
Abhay Shivayogimath (ㄱ) https://orcid.org/00000002-5152-0327

David M A Mackenzie (1) https://orcid.org/00000003-1114-2955

Bruno Dlubak (1) https://orcid.org/0000-0001-56968991

Birong Luo (1) https://orcid.org/0000-0003-0270-

0417

Cedric Huyghebaert (1) https://orcid.org/0000-00016043-7130

Stiven Forti (1) https://orcid.org/0000-0002-89393175

Camilla Coletti (1) https://orcid.org/0000-00028134-7633

Alba Centeno (이 https://orcid.org/0000-0001-84422283

Peter Bøggild (1) https://orcid.org/0000-0002-43420449

\section{References}

[1] Backes C et al 2020 Production and processing of graphene and related materials 2D Mater. 7022001

[2] Horng J et al 2011 Drude conductivity of Dirac fermions in graphene Phys. Rev. B 83 1-5

[3] Tomaino J L, Jameson A D, Kevek J W, Paul M J, Zande A M, van der Barton R A, McEuen P L, Minot E D and Lee Y-S 2011 Terahertz imaging and spectroscopy of large-area single-layer graphene Opt. Express 19 141-6

[4] Maeng I, Lim S, Chae S J, Lee Y H, Choi H and Son J H 2012 Gate-controlled nonlinear conductivity of Dirac fermion in graphene field-effect transistors measured by terahertz time-domain spectroscopy Nano Lett. 12 551-5

[5] Buron J D et al 2012 Graphene conductance uniformity mapping Nano Lett. 12 5074-81

[6] Buron J D, Mackenzie D M A, Petersen D H, Pesquera A, Centeno A, Bøggild P, Zurutuza A and Jepsen P U 2015 Terahertz wafer-scale mobility mapping of graphene on insulating substrates without a gate Opt. Express 233072

[7] Jnawali G, Rao Y, Yan H and Heinz T F 2013 Observation of a transient decrease in terahertz conductivity of single-layer graphene induced by ultrafast optical excitation Nano Lett. 13 524-30

[8] Whelan P R et al 2018 Electrical homogeneity mapping of epitaxial graphene on silicon carbide ACS Appl. Mater. Interfaces 10 31641-7

[9] Whelan P R, Zhao X, Pasternak I, Strupinski W, Jepsen P U and Bøggild P 2019 Non-contact mobility measurements of graphene on silicon carbide Microelectron. Eng. 212 9-12

[10] Mishra N et al 2019 Wafer-scale synthesis of graphene on sapphire: toward fab-compatible graphene Small 151904906

[11] Whelan P R et al 2018 Conductivity mapping of graphene on polymeric films by terahertz time-domain spectroscopy Opt. Express 26 17748-54

[12] Whelan P R et al 2020 Fermi velocity renormalization in graphene probed by terahertz time-domain spectroscopy 2D Mater. 7035009

[13] Ivanov I, Bonn M, Mics Z and Turchinovich D 2015 Perspective on terahertz spectroscopy of graphene Europhys. Lett. 11167001

[14] Bøggild P, Mackenzie D M A, Whelan P R, Petersen D H, Buron J D, Zurutuza A, Gallop J, Hao L and Jepsen P U 2017 Mapping the electrical properties of large-area graphene 2D Mater. 4042003

[15] Kužel P and Němec H 2020 Terahertz spectroscopy of nanomaterials: a close look at charge-carrier transport $A d v$. Opt. Mater. 81900623
[16] Hafez H A, Kovalev S, Tielrooij K, Bonn M, Gensch M and Turchinovich D 2020 Terahertz nonlinear optics of graphene: from saturable absorption to high-harmonics generation Adv. Opt. Mater. 81900771

[17] Docherty C J, Parkinson P, Joyce H J, Chiu M-H, Chen C-H, Lee M-Y, Li L-J, Herz L M and Johnston M B 2014 Ultrafast transient terahertz conductivity of monolayer $\mathrm{MoS}_{2}$ and $\mathrm{WSe}_{2}$ grown by chemical vapor deposition ACS Nano 8 11147-53

[18] Liu X, Yu H, Ji Q, Gao Z, Ge S, Qiu J, Liu Z, Zhang Y and Sun D 2016 An ultrafast terahertz probe of the transient evolution of the charged and neutral phase of photo-excited electron-hole gas in a monolayer semiconductor 2D Mater. 3014001

[19] Ji J, Zhou S, Wang W, Ling F and Yao J 2019 Active control of terahertz plasmon-induced transparency in the hybrid metamaterial/monolayer $\mathrm{MoS}_{2} / \mathrm{Si}$ structure Nanoscale 11 9429-35

[20] Shivayogimath A et al 2019 A universal approach for the synthesis of two-dimensional binary compounds Nat. Commun. 102957

[21] Tarekegne A T, Zhou B, Kaltenecker K, Iwaszczuk K, Clark S and Jepsen P U 2019 Terahertz time-domain spectroscopy of zone-folded acoustic phonons in $4 \mathrm{H}$ and 6H silicon carbide Opt. Express 273618

[22] Dai J, Xie X and Zhang X-C 2006 Detection of broadband terahertz waves with a laser-induced plasma in gases Phys. Rev. Lett. 97103903

[23] Wang T, Iwaszczuk K, Wrisberg E A, Denning E V and Jepsen P U 2016 Linearity of air-biased coherent detection for terahertz time-domain spectroscopy J. Infrared Millim. Terahertz Waves 37 592-604

[24] Strait J H, George P A, Dawlaty J, Shivaraman S, Chandrashekhar M, Rana F and Spencer M G 2009 Emission of terahertz radiation from SiC Appl. Phys. Lett. 95051912

[25] Grischkowsky D, Keiding S, Van Exter M and Fattinger C 1990 Far-infrared time-domain spectroscopy with terahertz beams of dielectrics and semiconductors J. Opt. Soc. Am. B 72006

[26] Whelan P R, Iwaszczuk K, Wang R, Hofmann S, Bøggild P and Jepsen P U 2017 Robust mapping of electrical properties of graphene from terahertz time-domain spectroscopy with timing jitter correction Opt. Express 252725

[27] Glover R E and Tinkham M 1957 Conductivity of superconducting films for photon energies between 0.3 and 40 kTc Phys. Rev. 108 243-56

[28] Gorecki J, Klokkou N, Piper L, Mailis S, Papasimakis N and Apostolopoulos V 2020 High-precision THz-TDS via self-referenced transmission echo method Appl. Opt. 596744

[29] Whelan P R, Shen Q, Luo D, Wang M, Ruoff R S, Jepsen P U, Bøggild P and Zhou B 2020 Reference-free THz-TDS conductivity analysis of thin conducting films Opt. Express 2828819

[30] Rehn A, Jahn D, Balzer J C and Koch M 2017 Periodic sampling errors in terahertz time-domain measurements Opt. Express 256712

[31] Kužel P, Němec H, Kadlec F, Kadlec C, Kuzel P, Nemec H, Kadlec F and Kadlec C 2010 Gouy shift correction for highly accurate refractive index retrieval in time-domain terahertz spectroscopy Opt. Express $1815338-48$

[32] Sensale-Rodriguez B, Yan R, Liu L, Jena D and Xing H G 2013 Graphene for reconfigurable THz optoelectronics Proc. IEEE 101 1705-16

[33] Buron J D et al 2014 Electrically continuous graphene from single crystal copper verified by terahertz conductance spectroscopy and micro four-point probe Nano Lett. 14 6348-55

[34] Strupinski W et al 2011 Graphene epitaxy by chemical vapor deposition on SiC Nano Lett. 11 1786-91 
[35] Stauber T, Peres N M R and Castro Neto A H 2008 Conductivity of suspended and non-suspended graphene at finite gate voltage Phys. Rev. B 78085418

[36] Hwang C, Siegel D A, Mo S-K, Regan W, Ismach A, Zhang Y, Zettl A and Lanzara A 2012 Fermi velocity engineering in graphene by substrate modification Sci. Rep. 2590

[37] González J, Guinea F and Vozmediano M A H 1994 Non-Fermi liquid behavior of electrons in the half-filled honeycomb lattice (A renormalization group approach) Nucl. Phys. B 424 595-618

[38] Das Sarma S, Hwang E H and Tse W-K 2007 Many-body interaction effects in doped and undoped graphene: Fermi liquid versus non-Fermi liquid Phys. Rev. B 75121406

[39] Stauber T, Parida P, Trushin M, Ulybyshev M V, Boyda D L and Schliemann J 2017 Interacting electrons in graphene: Fermi velocity renormalization and optical response Phys. Rev. Lett. 118266801

[40] Davies C L, Patel J B, Xia C Q, Herz L M and Johnston M B 2018 Temperature-dependent refractive index of quartz at terahertz frequencies J. Infrared Millim. Terahertz Waves 39 1236-48

[41] Cordon A et al $2019 \mathrm{THz}$ to inspect graphene and thin film materials 2019 44th Int. Conf. on Infrared, Millimeter, and Terahertz Waves (IRMMW-THz) (IEEE) pp 1-2

[42] Mackenzie D M A, Whelan P R, Bøggild P, Jepsen P U, Redo-Sanchez A, Etayo D, Fabricius N and Petersen D H 2018 Quality assessment of terahertz time-domain spectroscopy transmission and reflection modes for graphene conductivity mapping Opt. Express 26 9220-9

[43] Lin H, Braeuninger-Weimer P, Kamboj V S, Jessop D S, Degl'Innocenti R, Beere H E, Ritchie D A, Zeitler J A and Hofmann S 2017 Contactless graphene conductivity mapping on a wide range of substrates with terahertz time-domain reflection spectroscopy Sci. Rep. 7 1-9

[44] Lin H, Burton O J, Engelbrecht S, Tybussek K-H, Fischer B M and Hofmann S 2020 Through-substrate terahertz time-domain reflection spectroscopy for environmental graphene conductivity mapping Appl. Phys. Lett. 116021105

[45] Pizzocchero F et al 2015 Non-destructive electrochemical graphene transfer from reusable thin-film catalysts Carbon N. Y. 85 397-405

[46] Pandey H et al 2018 All CVD boron nitride encapsulated graphene FETs with CMOS compatible metal edge contacts IEEE Trans. Electron Devices 65 4129-34

[47] Melios C et al 2020 Towards standardisation of contact and contactless electrical measurements of CVD graphene at the macro-, micro- and nano-scale Sci. Rep. 103223

[48] Mackenzie D M A, Kalhauge K G, Whelan P R, Østergaard F W, Pasternak I, Strupinski W, Bøggild P, Jepsen P U and Petersen D H 2020 Wafer-scale graphene quality assessment using micro four-point probe mapping Nanotechnology 31225709

[49] Bøggild P, Grey F, Hassenkam T, Greve D R and Bjørnholm T 2000 Direct measurement of the microscale conductivity of conjugated polymer monolayers $A d v$. Mater. 12 947-50

[50] Tanikawa T, Matsuda I, Hobara R and Hasegawa S 2003 Variable-temperature micro-four-point probe method for surface electrical conductivity measurements in ultrahigh vacuum e-Journal Surf. Sci. Nanotechnol. 150-56

[51] Clarysse T et al 2006 Accurate sheet resistance measurement on ultra-shallow profiles Mater. Res. Soc. Symp. Proc. 912 197-202

[52] Petersen D H et al 2010 Review of electrical characterization of ultra-shallow junctions with micro four-point probes J. Vac. Sci. Technol. B 28 C1C27-33

[53] Walther M, Cooke D G, Sherstan C, Hajar M, Freeman M R and Hegmann F A 2007 Terahertz conductivity of thin gold films at the metal-insulator percolation transition Phys. Rev. B 76125408
[54] Němec H, Kužel P and Sundström V 2010 Charge transport in nanostructured materials for solar energy conversion studied by time-resolved terahertz spectroscopy J. Photochem. Photobiol. A 215 123-39

[55] Ulbricht R, Hendry E, Shan J, Heinz T F and Bonn M 2011 Carrier dynamics in semiconductors studied with time-resolved terahertz spectroscopy Rev. Mod. Phys. 83 543-86

[56] Joyce H J, Boland J L, Davies C L, Baig S A and Johnston M B 2016 A review of the electrical properties of semiconductor nanowires: insights gained from terahertz conductivity spectroscopy Semicond. Sci. Technol. 31103003

[57] Luo Y Y, Su F H, Zhang C, Zhong L, Pan S S, Xu S C, Wang H, Dai J M and Li G H 2017 Terahertz transport dynamics in the metal-insulator transition of $\mathrm{V} 2 \mathrm{O} 3$ thin film Opt. Commun. 387 385-9

[58] Isacsson A, Cummings A W, Colombo L, Colombo L, Kinaret J M and Roche S 2017 Scaling properties of polycrystalline graphene: a review 2D Mater. 4012002

[59] Yao W, Wu B and Liu Y 2020 Growth and grain boundaries in 2D materials ACS Nano 14 9320-46

[60] Smith N 2001 Classical generalization of the Drude formula for the optical conductivity Phys. Rev. B 64 1-6

[61] Cooke D G, Meldrum A and Uhd Jepsen P 2012 Ultrabroadband terahertz conductivity of Si nanocrystal films Appl. Phys. Lett. 101 1-5

[62] Dadrasnia E, Lamela H, Kuppam M B, Garet F and Coutaz J-L 2014 Determination of the DC electrical conductivity of multiwalled carbon nanotube films and graphene layers from noncontact time-domain terahertz measurements Adv. Condens. Matter Phys. 2014 1-6

[63] Pasternak I, Wesolowski M, Jozwik I, Lukosius M, Lupina G, Dabrowski P, Baranowski J M and Strupinski W 2016 Graphene growth on $\mathrm{Ge}(100) / \mathrm{Si}(100)$ substrates by CVD method Sci. Rep. 621773

[64] Pasternak I, Dabrowski P, Ciepielewski P, Kolkovsky V, Klusek Z, Baranowski J M and Strupinski W 2016 Large-area high-quality graphene on $\mathrm{Ge}(001) / \mathrm{Si}(001)$ substrates Nanoscale 8 11241-7

[65] Ciuk T, Pasternak I, Krajewska A, Sobieski J, Caban P, Szmidt J and Strupinski W 2013 Properties of chemical vapor deposition graphene transferred by high-speed electrochemical delamination J. Phys. Chem. C 117 20833-7

[66] Shimakawa K, Itoh T, Naito H and Kasap S O 2012 The origin of non-Drude terahertz conductivity in nanomaterials Appl. Phys. Lett. 100132102

[67] Lovrinčić R 2013 Comment on 'The origin of non-Drude terahertz conductivity in nanomaterials' [Appl. Phys. Lett. 100, 132102 (2012)] Appl. Phys. Lett. 102096101

[68] Shimakawa K, Itoh T, Naito H and Kasap S O 2013 Response to "Comment on 'The origin of non-Drude terahertz conductivity in nanomaterials"' [Appl. Phys. Lett. 102, 096101 (2013)] Appl. Phys. Lett. 102096102

[69] Cocker T L, Baillie D, Buruma M, Titova L V, Sydora R D, Marsiglio F and Hegmann F A 2017 Microscopic origin of the Drude-Smith model Phys. Rev. B 96205439

[70] Ostatnický T, Pushkarev V, Němec H and Kužel P 2018 Quantum theory of terahertz conductivity of semiconductor nanostructures Phys. Rev. B 97085426

[71] Cultrera A et al 2019 Mapping the conductivity of graphene with electrical resistance tomography Sci. Rep. 910655

[72] Boll M, Lotz M R, Hansen O, Wang F, Kjær D, Bøggild P and Petersen D H 2014 Sensitivity analysis explains quasi-one-dimensional current transport in two-dimensional materials Phys. Rev. B 90245432

[73] Lotz M R, Boll M, Hansen O, Kjær D, Bøggild P and Petersen D H 2014 Revealing origin of quasi-one dimensional current transport in defect rich two dimensional materials Appl. Phys. Lett. 105053115

[74] Lotz M R, Boll M, Østerberg F W, Hansen O and Petersen D H 2016 Mesoscopic current transport in 
two-dimensional materials with grain boundaries: four-point probe resistance and Hall effect J. Appl. Phys. 120134303

[75] Jessen B S, Whelan P R, Mackenzie D M A, Luo B, Thomsen J D, Gammelgaard L, Booth T J and Bøggild P 2018 Quantitative optical mapping of two-dimensional materials Sci. Rep. 86381

[76] Dai J, Zhang J, Zhang W and Grischkowsky D 2004 Terahertz time-domain spectroscopy characterization of the far-infrared absorption and index of refraction of high-resistivity, float-zone silicon J. Opt. Soc. Am. B 211379

[77] Luo D et al 2019 Adlayer-free large-area single crystal graphene grown on a $\mathrm{Cu}(111)$ foil Adv. Mater. 311903615

[78] Yu Q et al 2011 Control and characterization of individual grains and grain boundaries in graphene grown by chemical vapour deposition Nat. Mater. 10 443-9

[79] Huang P Y et al 2011 Grains and grain boundaries in single-layer graphene atomic patchwork quilts Nature 469 389-92

[80] Huang M et al 2018 Highly oriented monolayer graphene grown on a $\mathrm{Cu} / \mathrm{Ni}(111)$ alloy foil ACS Nano 12 6117-27

[81] Li B-W, Luo D, Zhu L, Zhang X, Jin S, Huang M, Ding F and Ruoff R S 2018 Orientation-dependent strain relaxation and chemical functionalization of graphene on a $\mathrm{Cu}(111)$ foil Adv. Mater. 301706504

[82] Nguyen V L et al 2015 Seamless stitching of graphene domains on polished copper (111) foil Adv. Mater. 27 1376-82

[83] Zhang J, Lin L, Jia K, Sun L, Peng H and Liu Z 2020 Controlled growth of single-crystal graphene films $A d v$. Mater. 321903266

[84] Xu X et al 2017 Ultrafast epitaxial growth of metre-sized single-crystal graphene on industrial Cu foil Sci. Bull. 62 1074-80

[85] Huang M et al 2020 Large-area single-crystal AB-bilayer and ABA-trilayer graphene grown on a $\mathrm{Cu} / \mathrm{Ni}(111)$ foil Nat. Nanotechnol. 15 289-95

[86] Jin S et al 2018 Colossal grain growth yields single-crystal metal foils by contact-free annealing Science 362 1021-5

[87] Levesque P L, Sabri S S, Aguirre C M, Guillemette J, Siaj M, Desjardins P, Szkopek T and Martel R 2011 Probing charge transfer at surfaces using graphene transistors Nano Lett. 11 132-7

[88] Gammelgaard L, Caridad J M, Cagliani A, Mackenzie D M A, Petersen D H, Booth T J and Bøggild P 2014 Graphene transport properties upon exposure to PMMA processing and heat treatments 2D Mater. 1035005

[89] Dean C R et al 2010 Boron nitride substrates for high-quality graphene electronics Nat. Nanotechnol. $5722-6$

[90] Jandhyala S et al 2012 Atomic layer deposition of dielectrics on graphene using reversibly physisorbed ozone ACS Nano $62722-30$

[91] Sagade A A, Neumaier D, Schall D, Otto M, Pesquera A, Centeno A, Elorza A Z and Kurz H 2015 Highly air stable passivation of graphene based field effect devices Nanoscale 7 3558-64

[92] Mzali S et al 2016 Stabilizing a graphene platform toward discrete components Appl. Phys. Lett. 109253110

[93] Wang L et al 2013 One-dimensional electrical contact to a two-dimensional material Science 342 614-7

[94] Emtsev K V et al 2009 Towards wafer-size graphene layers by atmospheric pressure graphitization of silicon carbide Nat. Mater. 8 203-7
[95] Lindvall N, Cole M T and Yurgens A 2011 Large-area uniform graphene-like thin films grown by chemical vapor deposition directly on silicon nitride Appl. Phys. Lett. 98252107

[96] Sun J, Lindvall N, Cole M T, Wang T, Booth T J, Bggild P, Teo K B K, Liu J and Yurgens A 2012 Controllable chemical vapor deposition of large area uniform nanocrystalline graphene directly on silicon dioxide J. Appl. Phys. 111044103

[97] Chen Z, Qi Y, Chen X, Zhang Y and Liu Z 2019 Direct CVD growth of graphene on traditional glass: methods and mechanisms Adv. Mater. 311803639

[98] Whelan P R et al 2017 Raman spectral indicators of catalyst decoupling for transfer of CVD grown 2D materials Carbon N. Y. 117 75-81

[99] Luo D et al 2017 Role of graphene in water-assisted oxidation of copper in relation to dry transfer of graphene Chem. Mater. 29 4546-56

[100] Verguts K et al 2017 Controlling water intercalation is key to a direct graphene transfer ACS Appl. Mater. Interfaces 937484-92

[101] Shivayogimath A et al 2019 Do-it-yourself transfer of large-area graphene using an office laminator and water Chem. Mater. 31 2328-36

[102] Das A et al 2008 Monitoring dopants by Raman scattering in an electrochemically top-gated graphene transistor Nat. Nanotechnol. 3 210-5

[103] Lee J E, Ahn G, Shim J, Lee Y S and Ryu S 2012 Optical separation of mechanical strain from charge doping in graphene Nat. Commun. 31024

[104] Rajab K Z, Naftaly M, Linfield E H, Nino J C, Arenas D, Tanner D, Mittra R and Lanagan M 2008 Broadband dielectric characterization of aluminum oxide $\left(\mathrm{Al}_{2} \mathrm{O}_{3}\right)$ J. Microelectron. Electron. Packag. 5 2-7

[105] Mackenzie D M A, Buron J D, Bøggild P, Jepsen P U and Petersen D H 2016 Contactless graphene conductance measurements: the effect of device fabrication on terahertz time-domain spectroscopy Int. J. Nanotechnol. 13591

[106] Gopalan K K, Paulillo B, Mackenzie D M A, Rodrigo D, Bareza N, Whelan P R, Shivayogimath A and Pruneri V 2018 Scalable and tunable periodic graphene nanohole arrays for mid-infrared plasmonics Nano Lett. 18 5913-8

[107] Rahimi S, Tao L, Chowdhury S F, Park S, Jouvray A Buttress S, Rupesinghe N, Teo K and Akinwande D 2014 Toward $300 \mathrm{~mm}$ wafer-scalable high-performance polycrystalline chemical vapor deposited graphene transistors ACS Nano 8 10471-9

[108] Dietz R J B, Vieweg N, Puppe T, Zach A, Globisch B, Göbel T, Leisching P and Schell M 2014 All fiber-coupled $\mathrm{THz}$-TDS system with $\mathrm{kHz}$ measurement rate based on electronically controlled optical sampling Opt. Lett. 396482

[109] Yahyapour M, Jahn A, Dutzi K, Puppe T, Leisching P, Schmauss B, Vieweg N and Deninger A 2019 Fastest thickness measurements with a terahertz time-domain system based on electronically controlled optical sampling Appl. Sci. 91283

[110] Bae S et al 2010 Roll-to-roll production of 30-inch graphene films for transparent electrodes Nat. Nanotechnol. 5 574-8

[111] Kobayashi T et al 2013 Production of a 100-m-long high-quality graphene transparent conductive film by roll-to-roll chemical vapor deposition and transfer process Appl. Phys. Lett. 102 1-5 